\title{
Novel Narrow-Band-Gap Conjugated Copolymers Containing Phenothiazine-Arylcyanovinyl Units for Organic Photovoltaic Cell Applications
}

\author{
KUANG-CHIEH LI, ${ }^{1}$ YING-CHAN HSU, ${ }^{2}$ JIANN-T'SUEN LIN, ${ }^{2}$ CHANG-CHUNG YANG, ${ }^{3}$ KUNG-HWA WEI, ${ }^{1}$ \\ HONG-CHEU LIN ${ }^{1}$ \\ ${ }^{1}$ Department of Materials Science and Engineering, National Chiao Tung University, Hsinchu, Taiwan, \\ Republic of China \\ ${ }^{2}$ Institute of Chemistry, Academia Sinica, Taipei, Taiwan, Republic of China \\ ${ }^{3}$ Energy and Environmental Laboratories, Industrial Technology Research Institute, Hsinchu, Taiwan, \\ Republic of China
}

Received 16 January 2008; accepted 23 February 2008

DOI: 10.1002 / pola.22715

Published online in Wiley InterScience (www.interscience.wiley.com).

\begin{abstract}
A series of novel narrow-band-gap copolymers (P1-P12) composed of alkyl-substituted fluorene (FO) units and six analogous mono- and bis(2-aryl-2-cyanovinyl)-10-hexylphenothiazine monomers (M1-M6) were synthesized by a palladiumcatalyzed Suzuki coupling reaction with two different feed in ratios of FO to M1-M6 (molar ratio $=3: 1$ and 1:1). The absorption spectra of polymers P1-P12 exhibited broad peaks located in the UV and visible regions from 400 to $800 \mathrm{~nm}$ with optical band gaps at 1.55-2.10 eV, which fit near the wavelength of the maximum solar photon reflux. Electrochemical experiments displayed that the reversible $\mathrm{p}$ - and $n$-doping processes of copolymers were partially reversible, and the proper HOMO/LUMO levels enabled a high photovoltaic open-circuit voltage. As blended with $[6,6]$-phenyl $\mathrm{C}_{61}$ butyric acid methyl ester (PCBM) as an electron acceptor in bulk heterojunction photovoltaic devices, narrow-band-gap polymers P1-P12 as electron donors showed significant photovoltaic performance which varied with the intramolecular donoracceptor interaction and their mixing ratios to PCBM. Under $100 \mathrm{~mW} / \mathrm{cm}^{2}$ of AM 1.5 white-light illumination, the device of copolymer P12 produced the highest preliminary result having an open-circuit voltage of $0.64 \mathrm{~V}$, a short-circuit current of 2.70 $\mathrm{mA} / \mathrm{cm}^{2}$, a fill factor of 0.29 , and an energy conversion efficiency of $0.51 \%$. (ㅇ 2008 Wiley Periodicals, Inc. J Polym Sci Part A: Polym Chem 46: 4285-4304, 2008

Keywords: conjugated polymers; copolymerization; heteroatom-containing polymers; narrow band gap polymers; phenothiazine derivatives; photovoltaic cells
\end{abstract}

\section{INTRODUCTION}

Because energy harvesting directly from sunlight by using photovoltaic cells (PVCs) is a very

Correspondence to: H. C. Lin (E-mail: linhc@cc.nctu. edu.tw)

Journal of Polymer Science: Part A: Polymer Chemistry, Vol. 46, 4285-4304 (2008) (C) 2008 Wiley Periodicals, Inc. important way to utilize renewable energy of the nature, especially for the development of organic solar cells, it has increasingly attracted intensified attention recently. ${ }^{1-3}$ Compared with inorganics, organic materials such as polymers have the benefits to allow the devices to be easily produced into light weight, large area, and flexible panels. For example, regioregular poly(3-hexylthiophene) and other polythiophene 
derivatives $^{4,5}$ have been extensively investigated over the last decade, resulting in PVCs with the values of energy conversion efficiency (ECE) between $3.0 \%$ and $5.0 \%$. Among several types of organic solar cell materials investigated so far, semiconducting conjugated polymers with electron donor-acceptor architectures are one of the most effective ways to build intramolecular charge transfer (ICT) interaction between the electron donor (D) and electron acceptor (A) units. $^{6-14}$ Conjugated D-A copolymers with strong ICT effects are promising materials for the development of high performance polymerbased PVCs due to the merits of narrow bandgaps, ${ }^{9-11}$ broad absorption bands extending into the near-infrared spectral range, efficient photoinduced charge transfer and separation, pronounced charge photogeneration and collection, and high mobility of ambipolar charge transport. $^{12-14}$

To date, the most widely used narrow-bandgap (NBG) conjugated copolymers are those containing aromatic heterocycles, such as benzothiadiazole-,${ }^{8-9,15-17}$ thienopyrazine-,${ }^{13,18-19}$ and dioxythiophene-based ${ }^{20,21}$ derivatives, as the electron-accepting moieties, and those bearing polyfluorene units, which can be easily modulated through syntheses to possess stable physical characters, as the electron-donating moieties in the $\pi$-conjugated D-A copolymer systems. Lately, Pei and coworkers ${ }^{15}$ reported low-bandgap copolymers containing poly(9,9-dioctylfluorene) and 2,7-diyl-alt-[4,7-bis(3-decyloxythien-2yl)-2,1,3-benzothiadiazole]-5',5"-diyl bearing alkoxy side chains on the thiophene units efficiently lowered the band gaps of the copolymers and enhanced charge transfer to electron acceptors. Besides, a APFO-Green5 D-A copolymer ${ }^{22}$ was also newly showed to have higher hole mobility and better device performance with different thicknesses of active layers and amounts of acceptors.

It is noticeable that a well-known design of the electron-withdrawing unit would be an arylsubstituted cyano or nitro group, because the cyano and nitro groups are among the most widespread electron withdrawing groups in organic chemistry. ${ }^{23}$ On the other hand, polymers and organic molecules ${ }^{24,25}$ containing phenothiazine units or their derivatives as the electrondonating moieties have lately attracted considerable research interests on account of the unique electro-optical properties originated from their electron-rich sulfur and nitrogen heteroatoms on the heterocyclic compounds, which can make these molecules potential materials for the applications of light-emitting diodes, ${ }^{26,27}$ photovoltaic devices, ${ }^{28,29}$ and organic field effect transistors. ${ }^{30}$ In the past years, various attempts to reduce the band gaps of conjugated polymers have been studied by constructing conjugated systems more planar which will increase the delocalization of $\pi$-electrons on the backbones, and thus to reduce the band gaps of the polymers. Another approach to a series of NBG conjugated heterocyclic main-chain polymers consist of electron-accepting units (A), such as cyano or nitro groups, and electron-donating units (D), such as thiophene, furan, or pyrrole functional blocks, to form resonance structures (i.e., D-A $\leftrightarrow$ $\left.\mathrm{D}^{+} \mathrm{A}^{-}\right)$in the backbones. ${ }^{31,32}$ Recently, only some copolymers reported by Shim and coworkers ${ }^{28}$ about the basic phenothiazine-based structure and then to extend the conjugation length by inserting phenylene and cyanovinylene functionalities for applications in red-emitting and photovoltaic devices. Although a large number of phenothiazine-based copolymers have been synthesized for applications in light-emitting devices, only a very small number of phenothiazinebased copolymers have investigated about the longer conjugated relationship with heterocycliccontaining structures and the applications for photovoltaic devices.

Based on this concept, the syntheses and characterization of NBG copolymers (P1-P12) that utilize the donor-acceptor approach to achieve absorption in the visible range of 400 $800 \mathrm{~nm}$ are reported. A series of NBG copolymers P1-P12 derived from 9,9-dihexylfluorene (FO) units and six phenothiazine-based heteroarylene-cyanovinylene monomers (M1-M6) were prepared by the palladium-catalyzed Suzuki cross-coupling reaction, and the feed in molar ratios of FO components are equal to 75 and $50 \%$. The PVC devices consisting of an active layer, which was made of composite thin films containing fluorene-phenothiazine (FO-PT) copolymers blended with a fullerene derivative, i.e., ${ }^{6,6}$-phenyl $\mathrm{C}_{61}$ butyric acid methyl ester (PCBM), show promising performance with the best ECE value up to $0.51 \%$ under AM1.5 solar simulator. In the present study, we have successfully synthesized a series of novel phenothiazine-based copolymers by incorporating different numbers of electron donors and acceptors, such as thiophene and cyano groups, respectively, with various ratios of phenothiazine (PT) 


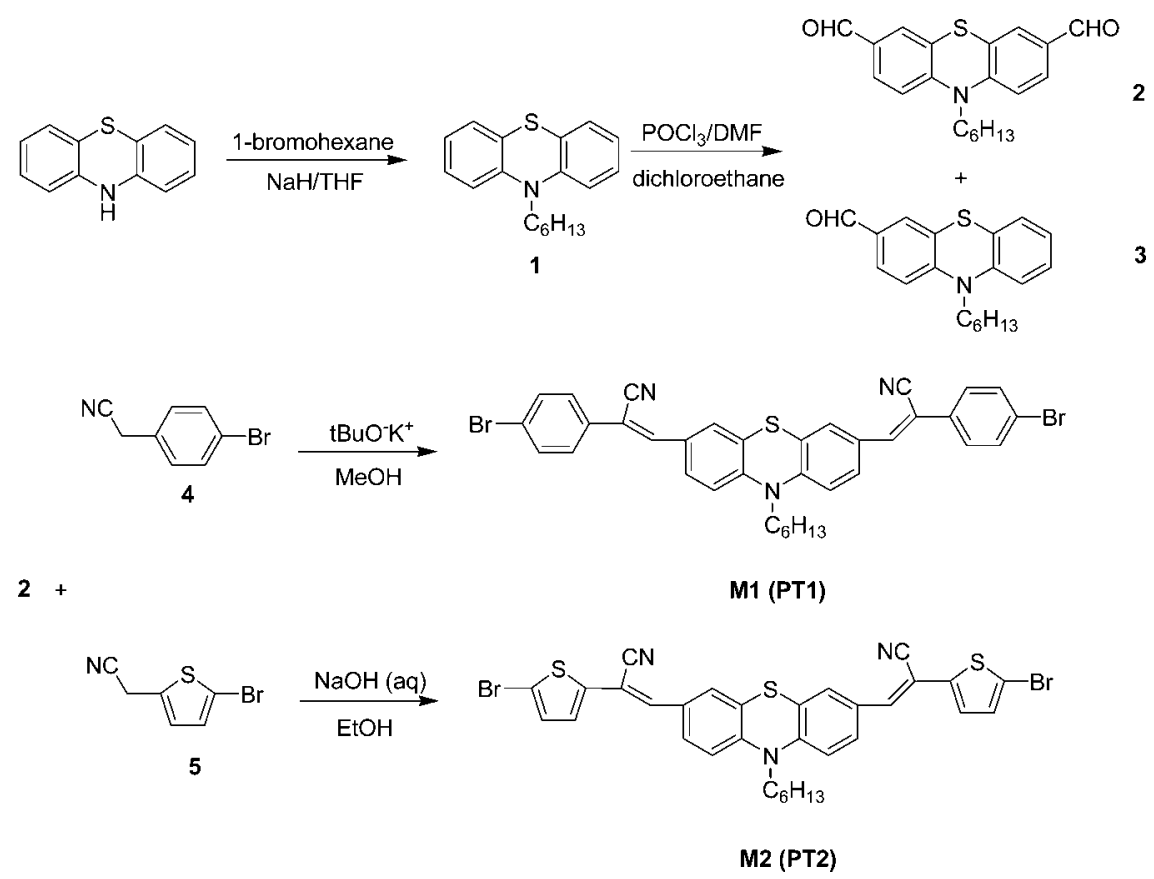

Scheme 1. Synthetic Routes of Symmetrical Monomers M1-M2.

units. The synthetic routes and structures of monomers M1-M6 and polymers P1-P12 are shown in Schemes 1-3. The optical and electronic properties, such as UV absorption spectra, electrochemical properties, photoluminescence quenching effects, and photovoltaic device results, of the copolymers are evaluated.

\section{EXPERIMENTAL}

\section{Materials}

Compounds 5-bromo-2-thiopheneacetonitrile (5), ${ }^{33}$ 2,7-dibromo-9,9-dihexylfluorene (11), ${ }^{34}$ and 2,7bis (4,4,5,5-tetramethyl-1,3,2-dioxaborolan-2-yl)9,9-dihexylfluorene $(\mathbf{1 2})^{35}$ were synthesized according to known literature procedures. The others precursors of monomers are shown in Schemes 1-3, and their synthetic procedures are describes as follows. Chemicals and solvents were reagent grades and purchased from Aldrich, ARCROS, TCI, and Lancaster Chemical Co. Dichloromathane and THF were distilled to keep anhydrous before use. All of the other chemicals and solvents were used as received.

\section{Synthesis}

The general synthetic procedures of all monomers M1-M6 (PT1-PT6) and polymers P1-P12 are described in Schemes 1-3, and their intermediates are described as follows:

\section{0-n-Hexylphenothiazine (1)}

In a flame-dried flask attached to a reflux condenser, $1.88 \mathrm{~g}$ of sodium hydride $(60 \%$ in mineral oil and washed by hexane, $78 \mathrm{mmol}$ ) was dissolved in $140 \mathrm{~mL}$ of anhydrous THF under nitrogen. $14.1 \mathrm{~g}$ (71 mmol) of phenothiazine dissolved in $80 \mathrm{~mL}$ of anhydrous THF was added into the clear solution. After refluxing for $1 \mathrm{~h}$, $15 \mathrm{~mL}$ of 1-bromohexane (106 mmol) was added. The mixture was refluxed for $24 \mathrm{~h}$ and then poured into $250 \mathrm{~mL}$ of water. The product was extracted with methylene chloride $(150 \mathrm{~mL} \times$ 3 , and the organic layer was dried over anhydrous magnesium sulfate. After purification by silica gel column chromatography with hexane as eluent, $16.9 \mathrm{~g}$ of colorless oil was obtained.

Yield: $85 \% .{ }^{1} \mathrm{H}$ NMR (DMSO- $d_{6}, \mathrm{ppm}$ ), $\delta: 7.16$ (m, $4 \mathrm{H}), 6.94(\mathrm{~m}, 4 \mathrm{H}), 3.83(\mathrm{t}, J=7.2 \mathrm{~Hz}, 2 \mathrm{H})$, $1.65(\mathrm{~m}, 2 \mathrm{H}), 1.40-1.15(\mathrm{~m}, 6 \mathrm{H}), 0.80(\mathrm{~m}, 3 \mathrm{H})$. ${ }^{13} \mathrm{C}$ NMR (DMSO- $\left.d_{6}, \mathrm{ppm}\right), \delta: 145.95,128.30$, $127.97,122.80,118.25,48.50,31.95,27.85$, $26.93,23.54,16.25$.

\section{0-n-Hexylphenothiazine-3,7-dicarbaldehyde (2)}

Compound 2 was synthesized by Vilsmeier formylation from Compound 1. A 500-mL three- 


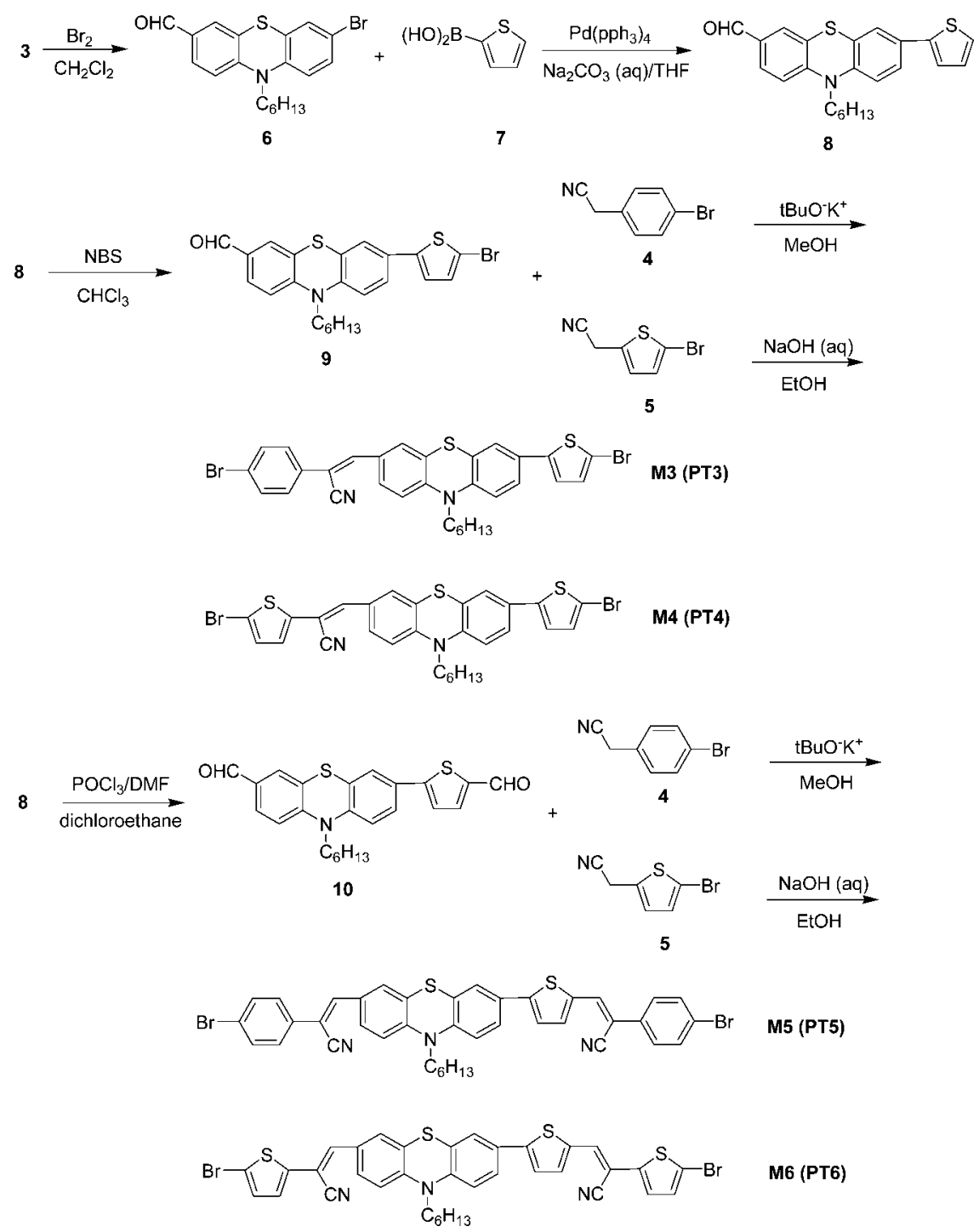

Scheme 2. Synthetic Routes of Asymmetrical Monomers M3-M6.

necked flask containing $20.04 \mathrm{~mL}(264 \mathrm{mmol})$ of anhydrous DMF was cooled in an ice bath. To the solution, $20.16 \mathrm{~mL}(216 \mathrm{mmol})$ of phosphorus chloride was added dropwisely for $30 \mathrm{~min}$. Compound 1 ( $6 \mathrm{~g}, 21.2 \mathrm{mmol})$ in $30 \mathrm{~mL}$ of $1,2-$ dichloroethane was added to the above solution and heated to $\sim 90{ }^{\circ} \mathrm{C}$ for 2 days. This solution was cooled to room temperature, poured into ice water, and neutralized to $\mathrm{pH}$ 6-7 by dropwise addition of saturated aqueous sodium hydroxide solution. The mixture was extracted with $\mathrm{CH}_{2} \mathrm{Cl}_{2} /$ water. The organic layer was dried with anhydrous $\mathrm{MgSO}_{4}$ and then concentrated under reduced pressure. The crude product was purified by column chromatography with ethyl acetate (EA)/hexane (1:4) to get $4.6 \mathrm{~g}$ of yellow solids.
Yield: $64 \% .{ }^{1} \mathrm{H}$ NMR (DMSO- $d_{6}$, ppm), $\delta: 9.79$ $(\mathrm{s}, 2 \mathrm{H}), 7.73(\mathrm{dd}, J=8.7,2.1 \mathrm{~Hz}, 2 \mathrm{H}), 7.60(\mathrm{~s}$, $2 \mathrm{H}), 7.21(\mathrm{~d}, J=8.4 \mathrm{~Hz}, 2 \mathrm{H}), 3.98(\mathrm{t}, J=7.2$ $\mathrm{Hz}, 2 \mathrm{H}), 1.67(\mathrm{~m}, 2 \mathrm{H}), 1.38-1.21(\mathrm{~m}, 6 \mathrm{H}), 0.80$ $(\mathrm{m}, 3 \mathrm{H}),{ }^{13} \mathrm{C}$ NMR (DMSO-d $\left.6, \mathrm{ppm}\right), \delta: 191.64$, $149.31,132.68,131.21,128.88,123.96,117.37$, 48.43, 31.72, 26.93, 26.57, 23.03, 14.79 .

\section{0-n-Hexylphenothiazine-3-carbaldehyde (3)}

The synthetic procedure of Compound $\mathbf{3}$ was similar to that of Compound $\mathbf{2}$, but the reactive time was just 1 day. The crude product was purified by column chromatography with EA/ hexane (1:6) to get $6.08 \mathrm{~g}$ of yellow solids.

Yield: $92 \% .{ }^{1} \mathrm{H}$ NMR (DMSO- $\left.d_{6}, \mathrm{ppm}\right), \delta: 9.77$ (s, $1 \mathrm{H}), 7.70(\mathrm{dd}, J=8.7,2.1 \mathrm{~Hz}, 1 \mathrm{H}), 7.58(\mathrm{~s}$,

Journal of Polymer Science: Part A: Polymer Chemistry DOI 10.1002/pola 
<smiles>CC(C)OB1OC(C)(C)C(C)(CC(C)(C)C)O1</smiles>

11<smiles>CC1(C)OB(c2ccc3c(c2)C([13CH2])([14CH2])c2cc(B4OC(C)(C)C(C)(C)O4)ccc2-3)OC1(C)C</smiles>

12<smiles>CC(C)(C)C1(C(=O)O)c2cc(Br)ccc2-c2ccc(Br)cc21</smiles>

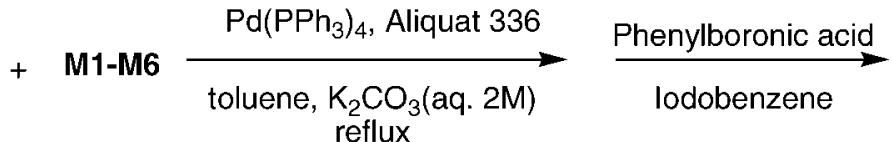<smiles>CC1(C)OB(c2ccc3c(c2)C([13CH2])([13CH2])c2cc(B4OC(C)(C)C(C)(C)O4)ccc2-3)OC1(C)C</smiles>

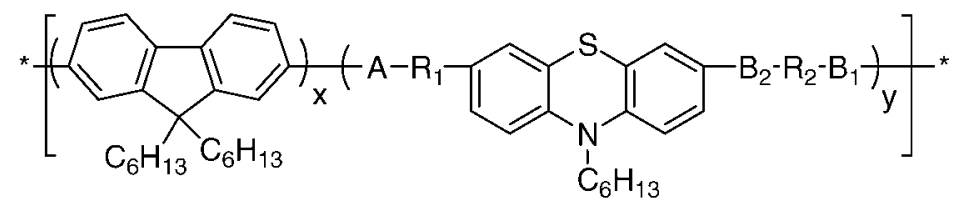

P1-P12

\begin{tabular}{|c|c|c|c|c|c|c|}
\hline polymers & $x: y$ & $-A-$ & $-\mathrm{R}_{1}-$ & $-\mathrm{B}_{2}-$ & $-\mathrm{R}_{2}-$ & $-\mathrm{B}_{1}-$ \\
\hline P1 & $3: 1$ & & $\mathrm{CN}$ & & & \\
\hline $\mathbf{P 2}$ & $1: 1$ & & & $X$ & & \\
\hline P3 & $3: 1$ & & $\mathrm{CN}$ & & & \\
\hline P4 & $1: 1$ & & & $X$ & & \\
\hline P5 & $3: 1$ & & $N$ & & & \\
\hline P6 & $1: 1$ & & & & $\mathrm{X}$ & $X$ \\
\hline P7 & $3: 1$ & & $N$ & & & \\
\hline P8 & $1: 1$ & & & & & \\
\hline P9 & $3: 1$ & & $\mathrm{CN}$ & & & \\
\hline P10 & $1: 1$ & & & & & \\
\hline P11 & $3: 1$ & & J & & & \\
\hline P12 & $1: 1$ & & & & & \\
\hline
\end{tabular}

Scheme 3. Synthetic Routes and Compositions (Molar Ratios) of Copolymers P1-P12.

1H), 7.21-6.98 (m, 5H), $3.92(\mathrm{t}, J=7.2 \mathrm{~Hz}, 2 \mathrm{H})$, $1.66(\mathrm{~m}, 2 \mathrm{H}), 1.36-1.21(\mathrm{~m}, 6 \mathrm{H}), 0.81(\mathrm{~m}, 3 \mathrm{H})$. ${ }^{13} \mathrm{C}$ NMR (DMSO- $\left.d_{6}, \mathrm{ppm}\right), \delta: 191.49,151.03$,
$143.96,131.79,131.12,128.89,128.71,128.22$, $124.57,124.54,123.53,117.48,116.47,47.94$, $31.75,27.03,26.66,23.02,14.77$.

Journal of Polymer Science: Part A: Polymer Chemistry DOI $10.1002 /$ pola 


\section{7-Bromo-10-hexyphenothiazine-3-carbaldehyde (6)}

Compound 3 (5.4 g, $17.3 \mathrm{mmol}$ ) was dissolved in $60 \mathrm{~mL}$ of dichloromethane and cooled to $5-10{ }^{\circ} \mathrm{C}$ with ice-water bath, and then $1.1 \mathrm{~mL}$ of bromine (20.8 mmol) in $5 \mathrm{~mL}$ of dichloromethane was added into the solution dropwise. The mixture was stirred for $2 \mathrm{~h}$ at room temperature. Saturated sodium hydrogen sulfite solution was added to the reaction mixture and stirred for $30 \mathrm{~min}$. After this, the reaction mixture was extracted with dichloromethane, dried over anhydrous magnesium sulfate, and purified by silica gel column flash chromatography with EA/hexane (1:6) as eluent. A pale yellow liquid was obtained.

Yield: $92 \% .{ }^{1} \mathrm{H}$ NMR (DMSO- $\left.d_{6}, \mathrm{ppm}\right), \delta: 9.78$ (s, 1H), $7.71(\mathrm{dd}, J=8.7,2.1 \mathrm{~Hz}, 1 \mathrm{H}), 7.57(\mathrm{~d}, J$ $=1.8 \mathrm{~Hz}, 1 \mathrm{H}), 7.35(\mathrm{~m}, 2 \mathrm{H}), 7.14(\mathrm{~d}, J=8.4 \mathrm{~Hz}$, $1 \mathrm{H}), 6.97$ (dd, $J=8.1,0.9 \mathrm{~Hz}, 1 \mathrm{H}), 3.88(\mathrm{t}, J=$ $7.2 \mathrm{~Hz}, 2 \mathrm{H}), 1.63(\mathrm{~m}, 2 \mathrm{H}), 1.21-1.14(\mathrm{~m}, 6 \mathrm{H})$, $0.79(\mathrm{~m}, 3 \mathrm{H}) .{ }^{13} \mathrm{C}$ NMR (DMSO- $\left.d_{6}, \mathrm{ppm}\right), \delta$ : $191.39,150.52,143.35,131.99,131.34,131.25$, $130.01,128.78,126.15,123.89,118.98,116.63$, 115.89, 48.04, 31.73, 26.89, 26.62, 23.02, 14.76.

\section{7-(Thiophen-2-yl)-10-hexylphenothiazine-3- carbaldehyde (8)}

Compound 6 (7.2 g, $18.4 \mathrm{mmol}$ ), thiophen-2-yl-2boronic acid (7) (3.1 g, $23.9 \mathrm{mmol})$, and tetrakis (triphenylphosphine)palladium $(0)(0.64 \mathrm{~g})$ were reacted in THF $(120 \mathrm{~mL})$ for $10 \mathrm{~min}$, and then $80 \mathrm{~mL}$ of $2 \mathrm{M}$ aqueous $\mathrm{Na}_{2} \mathrm{CO}_{3}$ solution was added. The reaction mixture was refluxed for 48 h. The cooled solution was washed with dilute hydrochloric acid (10\%) and water, and dried over magnesium sulfate. The final solution was purified by column chromatography (silica gel, $\mathrm{CH}_{2} \mathrm{Cl}_{2} /$ hexane 1:10) to yield a yellow solid.

Yield: $80 \%$. ${ }^{1} \mathrm{H}$ NMR $\left(\mathrm{CDCl}_{3}, \mathrm{ppm}\right), \delta: 9.78(\mathrm{~s}$, $1 \mathrm{H}), 7.61(\mathrm{~m}, 2 \mathrm{H}), 7.35(\mathrm{~m}, 2 \mathrm{H}), 7.25-7.19(\mathrm{~m}$, $2 \mathrm{H}), 7.04(\mathrm{~m}, 1 \mathrm{H}), 6.86(\mathrm{~m}, 2 \mathrm{H}), 3.87(\mathrm{t}, J=7.2$ $\mathrm{Hz}, 2 \mathrm{H}), 1.81(\mathrm{~m}, 2 \mathrm{H}), 1.46-1.24(\mathrm{~m}, 6 \mathrm{H}), 0.85$ $(\mathrm{m}, 3 \mathrm{H}),{ }^{13} \mathrm{C}$ NMR $\left(\mathrm{CDCl}_{3}, \mathrm{ppm}\right), \delta: 189.89$, $150.16,142.86,142.40,130.99,130.16,130.04$, $128.28,128.01,125.04,124.57,124.46,124.21$, $124.14,122.57,115.96,114.65,48.02,31.32$, $26.58,26.43,22.52,13.94$.

\section{7-(5-Bromothiophen-2-yl)-10-hexylphenothiazine- 3-carbaldehyde (9)}

Compound 8 ( $8.45 \mathrm{~g}, 21.47 \mathrm{mmol}$ ) was dissolved in dichloromethane $(50 \mathrm{~mL})$ under nitrogen, and $N$ bromosuccinimide (4.2 g, $23.61 \mathrm{mmol}$ ) was added all at once. After refluxing the reaction mixture for $1 \mathrm{~h}$, the product was poured into water $(200$ $\mathrm{mL})$. The solution was extracted with dichloromethane $(100 \mathrm{~mL} \times 3)$, and dried over magnesium sulfate. The solvent was removed under reduced pressure, and the crude product was purified by column chromatography on silica gel (EA/hexane 1:10) to afford Compound $\mathbf{1 2}(8.76 \mathrm{~g})$.

Yield: $86 \%$. ${ }^{1} \mathrm{H}$ NMR $\left(\mathrm{CDCl}_{3}, \mathrm{ppm}\right), \delta: 9.78(\mathrm{~s}$, $1 \mathrm{H}), 7.61(\mathrm{~m}, 2 \mathrm{H}), 7.24(\mathrm{~m}, 2 \mathrm{H}), 6.98-6.81(\mathrm{~m}$, $4 \mathrm{H}), 3.86(\mathrm{t}, J=7.2 \mathrm{~Hz}, 2 \mathrm{H}), 1.79(\mathrm{~m}, 2 \mathrm{H})$, 1.45-1.24 (m, 6H), $0.86(\mathrm{~m}, 3 \mathrm{H}) .{ }^{13} \mathrm{C} \quad \mathrm{NMR}$ $\left(\mathrm{CDCl}_{3}, \mathrm{ppm}\right), \delta: 189.81,152.13,148.89,144.36$, $141.75,136.53,131.34,130.79,129.11,128.34$, $125.56,124.34,124.13,123.77,123.45,115.83$, $114.65,48.13,31.45,26.64,26.46,22.53,13.67$.

\section{7-(5-Formylthiophen-2-yl)-10-hexylphenothiazine- 3-carbaldehyde (10)}

Compound 10 was synthesized by Vilsmeier formylation from Compound 8. A 500-mL threenecked flask containing $18.7 \mathrm{~mL}(240.9 \mathrm{mmol})$ of anhydrous DMF was cooled in an ice bath. To the solution, $18.4 \mathrm{~mL}$ (198 mmol) of phosphorus chloride was added dropwisely for $30 \mathrm{~min}$. Compound $1(13 \mathrm{~g}, 33.0 \mathrm{mmol})$ in $100 \mathrm{~mL}$ of 1,2 dichloroethane was added to the above solution and heated to $\sim 90{ }^{\circ} \mathrm{C}$ for 2 days. This solution was cooled to room temperature, poured into ice water, and neutralized to $\mathrm{pH}$ 6-7 by dropwise addition of saturated aqueous sodium hydroxide solution. The mixture was extracted with $\mathrm{CH}_{2} \mathrm{Cl}_{2}$ /water, and the organic layer was dried with anhydrous $\mathrm{MgSO}_{4}$ and then concentrated under reduced pressure. The crude product was purified by column chromatography with EA/ hexane (1:5) to get $8.0 \mathrm{~g}$ of yellow solids.

Yield: $58 \%$. ${ }^{1} \mathrm{H}$ NMR $\left(\mathrm{CDCl}_{3}, \mathrm{ppm}\right), \delta: 9.85(\mathrm{~s}$, $1 \mathrm{H}), 9.79(\mathrm{~s}, 1 \mathrm{H}), 7.70-7.55(\mathrm{~m}, 3 \mathrm{H}), 7.42-7.27$ $(\mathrm{m}, 3 \mathrm{H}), 6.86(\mathrm{~m}, 2 \mathrm{H}), 3.86(\mathrm{t}, J=7.2 \mathrm{~Hz}, 2 \mathrm{H})$, $1.77(\mathrm{~m}, 2 \mathrm{H}), 1.43-1.30(\mathrm{~m}, 6 \mathrm{H}), 0.88(\mathrm{~m}, 3 \mathrm{H})$. ${ }^{13} \mathrm{C}$ NMR $\left(\mathrm{CDCl}_{3}, \quad \mathrm{ppm}\right), \delta: 189.76,182.51$, $152.67,149.50,144.14,141.80,137.51,131.25$, $130.27,128.31,128.10,125.69,124.79,124.42$, $123.88,123.34,115.97,114.87,48.09,31.23$, $26.47,26.35,22.47,13.89$.

\section{General Procedures for the Syntheses of Monomers M1-M6 (PT1-PT6)}

There are two different synthetic routes of monomers M1-M6 (methods $\mathrm{A}^{11,33}$ and $\mathrm{B}^{36}$ ) as described as follows:

Journal of Polymer Science: Part A: Polymer Chemistry DOI 10.1002/pola 


\section{Method A}

A mixture of Compound 2 (or 12 or 13), Compound 4 (1-bromophenylacetonitrile), and methanol were placed in a $500-\mathrm{mL}$ two-neck roundbottom flask at room temperature. A catalytic amount of potassium tert-butoxide in methanol was added into this mixture. After $24 \mathrm{~h}$, the product was filtered and dried. The crude solid was further purified by column chromatography as outlined in the following text.

\section{Method B}

A mixture of Compound 2 (or 12 or 13) and Compound 5 (2-(5-bromothiophen-2-yl) acetonitrile) was dissolved in anhydrous ethanol under nitrogen in a $250-\mathrm{mL}$ two-necked round-bottomed flask. A mixture of sodium hydroxide and dry ethanol was added slowly, and then the crude product was precipitated in the reaction mixture. The reaction mixture was stirred for $24 \mathrm{~h}$ at room temperature, and the precipitate was filtered and washed with ethanol. The crude solid was further purified by column chromatography as outlined in the following text.

\section{M1 (PT1)}

Method A: Compound 2 (11.48 g, $33.8 \mathrm{mmol}$ ), Compound 4 (26.5 g, $135.2 \mathrm{mmol}$ ), methanol $(200 \mathrm{~mL})$, and a catalytic amount of potassium tert-butoxide were used. Chromatography on silica gel eluted with dichloromethane/hexane 3:2 afforded M1 as a red solid (18.36 g).

Yield: $78 \%$. ${ }^{1} \mathrm{H}$ NMR $\left(\mathrm{CDCl}_{3}, \mathrm{ppm}\right), \delta: 7.78$ (dd, $J=8.7,2.1 \mathrm{~Hz}, 2 \mathrm{H}), 7.55-7.47(\mathrm{~m}, 10 \mathrm{H})$, $7.31(\mathrm{~s}, 2 \mathrm{H}), 6.83(\mathrm{~d}, J=8.7 \mathrm{~Hz}, 2 \mathrm{H}), 3.83(\mathrm{t}$, $J=7.2 \mathrm{~Hz}, 2 \mathrm{H}), 1.80(\mathrm{~m}, 2 \mathrm{H}), 1.44-1.32(\mathrm{~m}, 6 \mathrm{H})$, $0.90(\mathrm{~m}, 3 \mathrm{H}) .{ }^{13} \mathrm{C} \mathrm{NMR}\left(\mathrm{CDCl}_{3}, \mathrm{ppm}\right), \delta: 145.58$, $140.59,133.44,132.12,128.78,128.40,128.28$, $127.17,123.54,123.00,117.91,115.24,107.89$, 48.10, 31.33, 26.47, 22.57, 22.10, 13.96. MS (EI): $\mathrm{m} / \mathrm{z}\left[\mathrm{M}^{+}\right]$695.04, calcd $\mathrm{m} / z\left[\mathrm{M}^{+}\right]$695.1. Anal. Calcd for $\mathrm{C}_{36} \mathrm{H}_{29} \mathrm{Br}_{2} \mathrm{~N}_{3} \mathrm{~S}$ : C, 62.17; H, 4.20; N, 6.04. Found: C, 62.35; H, 4.60; N, 6.39.

\section{M2 (PT2)}

Method B: Compound 2 (5.9 g, $17.35 \mathrm{mmol})$, Compound 5 (14.0 g, $69.4 \mathrm{mmol}$ ), sodium hydroxide (1.39 g, $34.7 \mathrm{mmol})$, and ethanol (130 $\mathrm{mL}$ ) were used. Chromatography on silica gel eluted with dichloromethane/hexane 1:3 afforded M2 as a red solid (7.5 g).

Yield: $61 \% .{ }^{1} \mathrm{H}$ NMR $\left(\mathrm{CDCl}_{3}, \mathrm{ppm}\right), \delta: 7.69$ (dd, $J=8.7,1.8 \mathrm{~Hz}, 2 \mathrm{H}), 7.38$ (s, 2H), 7.09-6.97 $(\mathrm{m}, 6 \mathrm{H}), 6.80(\mathrm{~d}, J=8.7 \mathrm{~Hz}, 2 \mathrm{H}), 3.80(\mathrm{t}, J=$ $7.2 \mathrm{~Hz}, 2 \mathrm{H}), 1.78(\mathrm{~m}, 2 \mathrm{H}), 1.41-1.23(\mathrm{~m}, 6 \mathrm{H})$, $0.88(\mathrm{~m}, 3 \mathrm{H}) .{ }^{13} \mathrm{C} \mathrm{NMR}\left(\mathrm{CDCl}_{3}, \mathrm{ppm}\right), \delta: 145.47$, $140.56,137.94,130.93,128.53,128.13,127.95$, $126.73,123.57,116.48,115.27,112.80,102.95$, 48.13, 31.34, 26.47, 22.58, 22.13, 13.97. MS (EI): $\mathrm{m} / \mathrm{z}\left[\mathrm{M}^{+}\right]$706.96, calcd $\mathrm{m} / z\left[\mathbf{M}^{+}\right]$707.1. Anal. Calcd for $\mathrm{C}_{32} \mathrm{H}_{25} \mathrm{Br}_{2} \mathrm{~N}_{3} \mathrm{~S}_{3}: \mathrm{C}, 54.32 ; \mathrm{H}, 3.56 ; \mathrm{N}$, 5.94. Found: C, 54.80; H, 4.03; N, 5.56.

\section{M3 (PT3)}

Method A: Compound 12 (8.76 g, $18.5 \mathrm{mmol})$, Compound 4 (14.5 g, $74.0 \mathrm{mmol}$ ), methanol (150 $\mathrm{mL}$ ), and a catalytic amount of potassium tertbutoxide were used. Chromatography on silica gel eluted with dichloromethane/hexane 1:5 afforded M3 as an orange solid (8.9 g).

Yield: $74 \% .{ }^{1} \mathrm{H}$ NMR $\left(\mathrm{CDCl}_{3}, \mathrm{ppm}\right), \delta: 7.80$ (dd, $J=8.7,2.1 \mathrm{~Hz}, 1 \mathrm{H}), 7.56-7.48(\mathrm{~m}, 4 \mathrm{H}), 7.32-7.22$ (m, 4H), 7.00-6.80 (m, 4H), $3.85(\mathrm{t}, J=7.2 \mathrm{~Hz}$, $2 \mathrm{H}), 1.80(\mathrm{~m}, 2 \mathrm{H}), 1.45-1.32(\mathrm{~m}, 6 \mathrm{H}), 0.89(\mathrm{~m}$, $3 \mathrm{H}) .{ }^{13} \mathrm{C} \mathrm{NMR}\left(\mathrm{CDCl}_{3}, \mathrm{ppm}\right), \delta: 146.58,144.47$, $143.16,140.82,133.59,132.09,130.82,128.75$, $128.45,127.75,127.14,124.72,124.14,123.81$, $122.87,122.54,118.01,115.61,115.01,110.77$, $107.41,47.85,31.36,26.56,26.50,22.57,13.97$. MS (EI): $m / z\left[\mathrm{M}^{+}\right] 649.99$, calcd $m / z\left[\mathrm{M}^{+}\right] 650.1$. Anal. Calcd for $\mathrm{C}_{31} \mathrm{H}_{26} \mathrm{Br}_{2} \mathrm{~N}_{2} \mathrm{~S}_{2}$ : C, 57.24; H, 4.03; N, 4.31. Found: C, 57.03; H, 4.43; N, 4.70.

\section{M4 (PT4)}

Method B: Compound 12 (2.88 g, $6.1 \mathrm{mmol}$ ), Compound 5 (4.9 g, $24.4 \mathrm{mmol})$, sodium hydroxide $(0.49 \mathrm{~g}, 12.2 \mathrm{mmol})$, and ethanol $(80 \mathrm{~mL})$ were used. Chromatography on silica gel eluted with EA/hexane 1:12 afforded M4 as a red solid (2.1 g).

Yield: $53 \% .{ }^{1} \mathrm{H} \mathrm{NMR}\left(\mathrm{CDCl}_{3}, \mathrm{ppm}\right), \delta: 7.72$ (dd, $J=8.7,1.8 \mathrm{~Hz}, 1 \mathrm{~Hz}), 7.47(\mathrm{~s}, 1 \mathrm{H}), 7.26-$ $7.21(\mathrm{~m}, 3 \mathrm{H}), 7.07-6.93(\mathrm{~m}, 5 \mathrm{H}), 6.82(\mathrm{~m}, 2 \mathrm{H})$, $3.84(\mathrm{t}, J=7.2 \mathrm{~Hz}, 2 \mathrm{H}), 1.80(\mathrm{~m}, 2 \mathrm{H}), 1.43-1.26$ $(\mathrm{m}, 6 \mathrm{H}), 0.89(\mathrm{~m}, 3 \mathrm{H}) .{ }^{13} \mathrm{C} \mathrm{NMR}\left(\mathrm{CDCl}_{3}, \mathrm{ppm}\right)$, $\delta: 146.53,144.47,143.12,140.69,138.24,130.89$, $130.81,128.77,128.59,128.13,127.39,126.55$, $124.72,124.15,123.88,122.55,116.58,115.60$, $115.03,112.58,110.78,102.49,47.87,31.36$, 26.56, 26.50, 22.57, 13.97. MS (EI): $\mathrm{m} / z\left[\mathrm{M}^{+}\right]$ 655.94, calcd $\mathrm{m} / \mathrm{z}\left[\mathrm{M}^{+}\right]$656.0. Anal. Calcd for 
$\mathrm{C}_{29} \mathrm{H}_{24} \mathrm{Br}_{2} \mathrm{~N}_{2} \mathrm{~S}_{3}:$ C， 53.05; H, 3.68; $\mathrm{N}, 4.27$. Found: C, 53.45; H, 4.03; N, 4.65.

\section{M5 (PT5)}

Method A: Compound 13 (2.5 g, 5.9 mmol), Compound 4 (4.7 g, $23.6 \mathrm{mmol})$, methanol $(100 \mathrm{~mL})$, and a catalytic amount of potassium tert-butoxide were used. Finally, a pure product can be obtained by recrystallization from $\mathrm{EA} /$ hexane 1:3 to afford M5 as a red solid (3.5 g).

Yield: $77 \%$. ${ }^{1} \mathrm{H}$ NMR $\left(\mathrm{CDCl}_{3}, \mathrm{ppm}\right), \delta: 7.79$ (dd, $J=8.7,2.1 \mathrm{~Hz}, 1 \mathrm{H}), 7.55-7.38(\mathrm{~m}, 12 \mathrm{H})$, $7.31(\mathrm{~m}, 2 \mathrm{H}), 7.20(\mathrm{~m}, 1 \mathrm{H}), 6.81(\mathrm{~m}, 2 \mathrm{H}), 3.82(\mathrm{t}$, $J=7.2 \mathrm{~Hz}, 2 \mathrm{H}), 1.79(\mathrm{~m}, 2 \mathrm{H}), 1.32(\mathrm{~m}, 6 \mathrm{H}), 0.88$ $(\mathrm{m}, 3 \mathrm{H}) .{ }^{13} \mathrm{C} \mathrm{NMR}\left(\mathrm{CDCl}_{3}, \mathrm{ppm}\right), \delta: 148.19$, $146.11,143.77,140.69,136.18,134.87,134.30$, $133.50,132.84,132.12,132.08,128.76,128.40$, $128.13,127.85,127.11,126.87,125.34,124.40$, $123.95,123.49,122.88,122.81,122.78,117.99$, $117.93,115.47,115.00,107.44,105.57,47.95$, 31.34, 26.62, 26.47, 22.57, 13.97. MS (EI): $\mathrm{m} / \mathrm{z}$ $\left[\mathrm{M}^{+}\right]$777.03, calcd $\mathrm{m} / z\left[\mathrm{M}^{+}\right]$777.1. Anal. Calcd for $\mathrm{C}_{40} \mathrm{H}_{31} \mathrm{Br}_{2} \mathrm{~N}_{3} \mathrm{~S}_{2}$ : C, 61.78; $\mathrm{H}, 4.02 ; \mathrm{N}, 5.40$. Found: C, 61.30; H, 4.52; N, 5.71.

\section{M6 (PT6)}

Method B: Compound 13 (2.0 g, $4.8 \mathrm{mmol})$, Compound 5 ( $3.8 \mathrm{~g}, 18.9 \mathrm{mmol}$ ), sodium hydroxide $(0.38 \mathrm{~g}, 9.6 \mathrm{mmol})$, and ethanol $(70 \mathrm{~mL})$ were used. Chromatography on silica gel eluted with dichloromethane/hexane 1:5 afforded $\mathbf{M 6}$ as a red solid $(2.1 \mathrm{~g})$.

Yield: $53 \% .{ }^{1} \mathrm{H}$ NMR $\left(\mathrm{CDCl}_{3}, \mathrm{ppm}\right), \delta: 7.72$ $(\mathrm{dd}, J=8.7,1.8 \mathrm{~Hz}, 1 \mathrm{H}), 7.45-7.36(\mathrm{~m}, 3 \mathrm{H})$, 7.29-7.18 (m, 3H), 7.07-7.01 (m, 5H), $6.79(\mathrm{~d}$, $J=8.7 \mathrm{~Hz}, 2 \mathrm{H}), 3.80(\mathrm{t}, J=7.2 \mathrm{~Hz}, 2 \mathrm{H}), 1.80(\mathrm{~m}$, $2 \mathrm{H}), 1.45-1.14(\mathrm{~m}, 6 \mathrm{H}), 0.89(\mathrm{~m}, 3 \mathrm{H}) .{ }^{13} \mathrm{C} \mathrm{NMR}$ $\left(\mathrm{CDCl}_{3}, \mathrm{ppm}\right), \delta: 148.11,146.00,143.66,140.67$, $140.14,138.08,135.80,134.54,132.05,131.00$, $130.89,130.67,128.54,128.11,127.49,126.56$, $125.31,124.32,123.88,123.53,122.87,116.56$, $116.49,115.43,114.99,112.64,112.62,102.51$, 100.94, 48.00, 31.35, 29.67, 26.49, 22.59, 13.98 . MS (EI): $m / z\left[\mathrm{M}^{+}\right]$788.94, calcd $m / z\left[\mathrm{M}^{+}\right]$789.0. Anal. Calcd for $\mathrm{C}_{36} \mathrm{H}_{27} \mathrm{Br}_{2} \mathrm{~N}_{3} \mathrm{~S}_{4}: \mathrm{C}, 54.75 ; \mathrm{H}$, 3.45; N, 5.32. Found: C, 55.19; H, 3.90; N, 4.82.

\section{General Procedures for the Syntheses of Copolymers P1-P12}

The synthetic route of polymers is shown in Scheme 3 . All of the polymerizations were car- ried out through the palladium(0)-catalyzed Suzuki coupling reactions. Into $50 \mathrm{~mL}$ of twoneck flask, 1 equiv. of dibromo compounds [(2,7dibromo-9,9-dihexylfluorene (11) and monomers M1-M6 (PT1-PT6)] and 1 equiv. of 2,7-bis[(4,4,5,5-tetramethyl-1,3,2-dioxaborolan-2-yl)-9,9dihexylfluorene (12)] were added in $10 \mathrm{~mL}$ of anhydrous toluene. The $\operatorname{Pd}(0)$ complex, $\operatorname{Pd}\{\mathrm{P}$ (p-tolyl)3\}3 (1 mol \%), was transferred into the mixture in a dry environment. Then, $2 \mathrm{M}$ aqueous potassium carbonate and the phase transfer catalyst, i.e., aliquat 336 (several drops), were subsequently transferred via cannula into the previous mixture under nitrogen. The reaction mixture was stirred at $90{ }^{\circ} \mathrm{C}$ for 2 days, and then the excess amount of iodobenzene and phenylboronic acid, the end-capper, dissolved in 1 $\mathrm{mL}$ of anhydrous toluene was added and stirring for $4 \mathrm{~h}$, respectively. The reaction mixture was cooled to $50{ }^{\circ} \mathrm{C}$ and added slowly into a vigorously stirred mixture of $300 \mathrm{~mL}$ of methanol. The polymers were collected by filtration and reprecipitation from methanol. The crude polymers were further purified by washing with acetone for 3 days in a Soxhlet apparatus to remove oligomers and catalyst residues. The resulting polymers were soluble in common organic solvents.

\section{$\mathrm{P1}\left(\mathrm{FO}_{3}-\mathrm{PT1}\right)$}

Compounds 11 (0.25 equiv.), 12 (0.5 equiv.), and M1 ( 0.25 equiv.) were used in this polymerization.

Yield: $62 \% .{ }^{1} \mathrm{H} \mathrm{NMR}\left(\mathrm{CDCl}_{3}, \mathrm{ppm}\right), \delta: 8.05-7.3$ (m, $32 \mathrm{H}, \mathrm{ArH}), 6.94$ (br, $2 \mathrm{H}$, vinylic proton), 3.89 (weak br, $\mathrm{CH}_{2}$ attached to nitrogen of phenothiazine, $2 \mathrm{H}), 2.06-0.76(\mathrm{~m}$, aliphatic, $\sim 89 \mathrm{H})$. ELEM. ANAL. Found: C, 85.05; H, 8.02; N, 2.75. S, 2.18.

\section{P2 (FO, - PT1)}

Compounds 12 (0.5 equiv.) and M1 (0.5 equiv.) were used in this polymerization.

Yield: $88 \% .{ }^{1} \mathrm{H}$ NMR $\left(\mathrm{CDCl}_{3}, \mathrm{ppm}\right), \delta: 7.90-7.32$ (m, 20H, ArH), 6.97 (br, $2 \mathrm{H}$, vinylic proton), 3.90 (weak br, $\mathrm{CH}_{2}$ attached to nitrogen of phenothiazine, $2 \mathrm{H}), 2.17-0.76$ (m, aliphatic, $\sim 37 \mathrm{H})$. Elem. ANAL. Found: C, 77.88; H, 6.71; N, 4.13. S, 3.30.

\section{$\mathrm{P3}$ (FO $\mathrm{F}_{3}$-PT2)}

Compounds 11 ( 0.25 equiv.), $\mathbf{1 2}$ (0.5 equiv.), and M2 $(0.25$ equiv.) were used in this polymerization.

Journal of Polymer Science: Part A: Polymer Chemistry 
Yield: $78 \% .{ }^{1} \mathrm{H}$ NMR $\left(\mathrm{CDCl}_{3}, \mathrm{ppm}\right), \delta: 7.83-$ 7.23 (m, 28H, ArH), 6.90 (br, $2 \mathrm{H}$, vinylic proton), 3.90 (weak br, $\mathrm{CH}_{2}$ attached to nitrogen of phenothiazine, $2 \mathrm{H}$ ), $2.20-0.77$ (m, aliphatic, $\sim 89 \mathrm{H}$ ). Elem. Anal. Found: C, 81.11; H, 7.71; N, 2.55. S, 6.00 .

\section{P4 (FO 1 -PT2)}

Compound 12 (0.5 equiv.) and M2 (0.5 equiv.) were used in this polymerization.

Yield: $61 \%$. ${ }^{1} \mathrm{H}$ NMR $\left(\mathrm{CDCl}_{3}, \mathrm{ppm}\right), \delta: 7.89$ 7.27 (m, 16H, ArH), 6.97 (br, $2 \mathrm{H}$, vinylic proton), 3.89 (weak br, $\mathrm{CH}_{2}$ attached to nitrogen of phenothiazine, $2 \mathrm{H}), 2.23-0.78(\mathrm{~m}$, aliphatic, $\sim 37 \mathrm{H})$. Elem. Anal. Found: C, 72.77; H, 6.34; N, 4.21. S, 9.72 .

\section{$\mathrm{P5}\left(\mathrm{FO}_{3}-\mathrm{PT} 3\right)$}

Compounds 11 ( 0.25 equiv.), 12 (0.5 equiv.), and M3 ( 0.25 equiv.) were used in this polymerization.

Yield: $82 \%$. ${ }^{1} \mathrm{H}$ NMR $\left(\mathrm{CDCl}_{3}, \mathrm{ppm}\right), \delta: 7.82-$ $7.25(\mathrm{~m}, 30 \mathrm{H}, \mathrm{ArH}), 6.90$ (br, $1 \mathrm{H}$, vinylic proton), 3.90 (weak br, $\mathrm{CH}_{2}$ attached to nitrogen of phenothiazine, $2 \mathrm{H}$ ), 2.17-0.77 (m, aliphatic, $\sim 89 \mathrm{H})$. Elem. Anal. Found: C, 81.58; H, 7.93; N, 1.88. S, 4.32 .

\section{$P 6$ (FO, - PT3)}

Compound 12 (0.5 equiv.) and M3 (0.5 equiv.) were used in this polymerization.

Yield: $64 \%$. ${ }^{1} \mathrm{H}$ NMR $\left(\mathrm{CDCl}_{3}, \mathrm{ppm}\right), \delta: 7.89-$ $7.26(\mathrm{~m}, 17 \mathrm{H}, \mathrm{ArH}), 6.88$ (br, $2 \mathrm{H}$, vinylic proton), 3.88 (weak br, $\mathrm{CH}_{2}$ attached to nitrogen of phenothiazine, $2 \mathrm{H}), 2.17-0.74(\mathrm{~m}$, aliphatic, $\sim 37 \mathrm{H})$. Elem. ANal. Found: C, 79.32; H, 7.13; N, 3.29. S, 7.30.

\section{$\mathrm{P7}\left(\mathrm{FO}_{3}-\mathrm{PT} 4\right)$}

Compounds 11 ( 0.25 equiv.), 12 ( 0.5 equiv.), and M4 (0.25 equiv.) were used in this polymerization.

Yield: $86 \%$. ${ }^{1} \mathrm{H}$ NMR $\left(\mathrm{CDCl}_{3}, \mathrm{ppm}\right), \delta: 7.83-$ $7.24(\mathrm{~m}, 28 \mathrm{H}, \mathrm{ArH}), 6.88$ (br, $1 \mathrm{H}$, vinylic proton), 3.89 (weak br, $\mathrm{CH}_{2}$ attached to nitrogen of phenothiazine, $2 \mathrm{H}$ ), $2.16-0.78$ (m, aliphatic, $\sim 89 \mathrm{H}$ ). Elem. Anal. Found: C, 81.41; H, 7.87; N, 1.77. S, 6.53 .

Journal of Polymer Science: Part A: Polymer Chemistry DOI 10.1002/pola

\section{$\mathrm{P8}\left(\mathrm{FO}_{1}\right.$-PT4)}

Compound 12 (0.5 equiv.) and M4 (0.5 equiv.) were used in this polymerization.

Yield: $71 \% .{ }^{1} \mathrm{H}$ NMR $\left(\mathrm{CDCl}_{3}, \mathrm{ppm}\right), \delta: 7.79$ 7.13 (m, 15H, ArH), 6.86 (br, $2 \mathrm{H}$, vinylic proton), 3.88 (weak br, $\mathrm{CH}_{2}$ attached to nitrogen of phenothiazine, $2 \mathrm{H}), 2.17-0.74(\mathrm{~m}$, aliphatic, $\sim 37 \mathrm{H})$. Elem. Anal. Found: C, 71.62; H, 6.30; N, 3.23. S, 11.38.

\section{$\mathrm{P9}\left(\mathrm{FO}_{3}\right.$-PT5)}

Compounds 11 ( 0.25 equiv.), 12 ( 0.5 equiv.), and M5 (0.25 equiv.) were used in this polymerization.

Yield: $87 \%$. ${ }^{1} \mathrm{H}$ NMR $\left(\mathrm{CDCl}_{3}, \mathrm{ppm}\right), \delta: 7.83-$ 7.25 (m, 34H, ArH), 6.90 (br, $2 \mathrm{H}$, vinylic proton), 3.90 (weak br, $\mathrm{CH}_{2}$ attached to nitrogen of phenothiazine, $2 \mathrm{H}), 2.17-0.77(\mathrm{~m}$, aliphatic, $\sim 89 \mathrm{H})$. Elem. Anal. Found: C, 83.24; H, 7.66; N, 2.47. S, 4.34 .

\section{P10 (FO, - PT5)}

Compound 12 (0.5 equiv.) and M5 (0.5 equiv.) were used in this polymerization.

Yield: $70 \%$. ${ }^{1} \mathrm{H}$ NMR $\left(\mathrm{CDCl}_{3}, \mathrm{ppm}\right), \delta: 7.80$ 7.08 (m, 22H, ArH), 6.86 (br, $2 \mathrm{H}$, vinylic proton), 3.87 (weak br, $\mathrm{CH}_{2}$ attached to nitrogen of phenothiazine, $2 \mathrm{H}), 2.17-0.76(\mathrm{~m}$, aliphatic, $\sim 37 \mathrm{H})$. Elem. ANal. Found: C, 80.48; H, 6.08; N, 4.31. S, 6.85 .

\section{$\mathrm{P11}\left(\mathrm{FO}_{3}\right.$-PT6)}

Compounds 11 ( 0.25 equiv.), 12 ( 0.5 equiv.), and M6 ( 0.25 equiv.) were used in this polymerization.

Yield: $89 \%$. ${ }^{1} \mathrm{H}$ NMR $\left(\mathrm{CDCl}_{3}, \mathrm{ppm}\right), \delta: 7.83-$ $7.24(\mathrm{~m}, 30 \mathrm{H}, \mathrm{ArH}), 6.86$ (br, $2 \mathrm{H}$, vinylic proton), 3.87 (weak br, $\mathrm{CH}_{2}$ attached to nitrogen of phenothiazine, $2 \mathrm{H}), 2.16-0.78(\mathrm{~m}$, aliphatic, $\sim 89 \mathrm{H})$. Elem. Anal. Found: C, 75.77; H, 7.12; N, 2.35. S, 7.46.

\section{P12 (FO -PT6)}

Compound 12 (0.5 equiv.) and $\mathbf{M 6}$ (0.5 equiv.) were used in this polymerization.

Yield: $75 \%$. ${ }^{1} \mathrm{H}$ NMR $\left(\mathrm{CDCl}_{3}, \mathrm{ppm}\right), \delta: 7.85-$ 7.27 (m, 18H, ArH), 6.89 (br, $2 \mathrm{H}$, vinylic proton), 3.87 (weak br, $\mathrm{CH}_{2}$ attached to nitrogen of phenothiazine, $2 \mathrm{H}), 2.14-0.77$ (m, aliphatic, $\sim 37 \mathrm{H}$ ). Elem. Anal. Found: C, 74.32; H, 6.07; N, 4.25. S, 13.29 . 


\section{Measurements and Characterization}

${ }^{1} \mathrm{H}$ NMR spectra were recorded on a Varia Unity $300 \mathrm{MHz}$ spectrometer using $\mathrm{CDCl}_{3}$ and DMSO solvents. Elemental analyses were performed on a HERAEUS CHN-OS RAPID elemental analyzer. Transition temperatures were determined by differential scanning calorimetry (DSC) on a Perkin-Elmer Pyris 7 thermal analyzer with a heating and cooling rate of $10{ }^{\circ} \mathrm{C} / \mathrm{min}$. Thermogravimetric analysis (TGA) was conducted with a TA instrument Q500 at a heating rate of $20^{\circ} \mathrm{C} /$ min under nitrogen. Gel permeation chromatography (GPC) analyses were conducted on a Waters 1515 separation module using polystyrene as a standard and THF as an eluent. UVvisible absorption spectra were recorded in dilute THF solutions $\left(10^{-6} \mathrm{M}\right)$ on a HP G1103A spectrophotometer. Thin films of UV-vis measurements were spin-coated on a quartz substrate from chlorobenzene solutions with a concentration of $5 \mathrm{mg} / \mathrm{mL}$. Electrochemistry measurements were performed using an Autolab Model PGSTAT30 potentiostat/galvanostat with a standard three-electrode electrochemical cell in a $0.1 \mathrm{M}$ tetrabutylammonium hexafluorophosphate (TBAPF6) solution (in acetonitrile) at room temperature with a scanning rate of 50 $\mathrm{mV} / \mathrm{s}$. A platinum working electrode, a platinum wire counter electrode, and an $\mathrm{Ag} / \mathrm{AgCl}$ reference electrode were used. The films of the polymers were coated onto the Pt working electrode by dipping the Pt wire into 1 wt \% THF solutions. The onset potentials were determined from the intersection of two tangents drawn at the rising current and background current of the cyclic voltammogram (CV).

\section{Device Fabrication and Characterization of PVCs}

The PVC device structure used in this study was a sandwich configuration of ITO/PEDOT: $\mathrm{PSS} /$ active layer/LiF/Al. We fabricated the PVC devices according to the procedures similar to those of electroluminescence devices. After drying the substrate of ITO, a thin layer $(\sim 50 \mathrm{~nm})$ of PEDOT:PSS was spin-coated and dried. Subsequently, on the top of the PEDOT:PSS layer, the active layer was prepared by spin coating from composite solutions of P2, P6, P8, and P10/PCBM (1:4 w/w) and of P12/PCBM (1:1, $1: 2$, and $1: 4 \mathrm{w} / \mathrm{w}$ ) in the mixed solvents of chlorobenzene and chloroform (1:1 vol). The spin rate was $\sim 800 \mathrm{rpm}$, and the thickness of the active layer was typically $\sim 100 \mathrm{~nm}$. The devices were completed by deposition with $1 \mathrm{~nm}$ of $\mathrm{LiF}$ and $150 \mathrm{~nm}$ of $\mathrm{Al}$. For PVC measurements, $I-V$ curves were recorded under a solar simulator with AM 1.5 illumination (at $100 \mathrm{~mW} / \mathrm{cm}^{2}$ ). All cells were prepared and measured under ambient conditions.

\section{RESULTS AND DISCUSSION}

\section{Synthesis and Characterization}

The general synthetic routes of monomers M1M6 (PT1-PT6) are shown in Schemes 1-2. All monomers were synthesized by following the modified multistep procedures in the literature, ${ }^{40,41}$ and some of them (compounds 2, 3, and 6 ) were started from commercially available phenothiazine. Monomers (M1-M6 and PT1PT6) were satisfactorily characterized by ${ }^{1} \mathrm{H}$ NMR, ${ }^{13} \mathrm{C}$ NMR, MS spectroscopy, and elemental analyses. The synthetic procedures towards polymers P1-P12 are outlined in Scheme 3. Conjugated polymers derived from monomers M1-M6 were prepared by palladium(0)-catalyzed Suzuki coupling reactions with an equivalent molar ratio of diboronic ester monomer 12 to dibromo monomers (11 and M1-M6). During the polymerization, the feed in monomer ratios of fluorene units to monomers M1-M6 were 3:1 and 1:1, respectively. The copolymers P1-P12 were synthesized from a Suzuki coupling reaction in a biphasic system (toluene/aqueous $\left.\mathrm{Na}_{2} \mathrm{CO}_{3}\right)$ with freshly prepared $\mathrm{Pd}\left\{\mathrm{P}(p \text {-tolyl })_{3}\right\}_{3}$ as a catalyst precursor. ${ }^{42}$ The obtained polymers were further purified by washing with acetone in a Soxhlet apparatus for $24 \mathrm{~h}$ to remove oligomers and catalyst residues and were dried under reduced pressure at room temperature. After purification and drying, all polymers were obtained as red fibrous solids in overall good yields (61-89\%). As shown in Table 1, the elemental analyses indicated that the FO contents in copolymers P1-P12 were very close to the feed in compositions. All copolymers exhibited good solubilities in common organic solvents, such as THF, chloroform, and chlorobenzene. The molecular weights of the polymers determined by GPC against polystyrene standards in THF are summarized in Table 1 . These results show that considerable molecular weights were obtained in these copolymers, which had number-average molecular weights $\left(M_{\mathrm{n}}\right)$ ranging 
Table 1. Molecular Weights, Yields, and Thermal Data of Polymers and PT Content in the Copolymers

\begin{tabular}{|c|c|c|c|c|c|c|c|}
\hline \multirow[b]{2}{*}{ Polymer } & \multirow[b]{2}{*}{$M_{\mathrm{w}}^{\mathrm{a}}$} & \multirow[b]{2}{*}{$\mathrm{PDI}^{\mathrm{a}}$} & \multicolumn{2}{|c|}{ Ratio of PT Units } & \multirow[b]{2}{*}{$\begin{array}{c}\text { Yield } \\
(\%)\end{array}$} & \multirow[b]{2}{*}{$\begin{array}{c}T_{\mathrm{g}}^{\mathrm{c}} \\
\left({ }^{\circ} \mathrm{C}\right)\end{array}$} & \multirow[b]{2}{*}{$\begin{array}{l}T_{\mathrm{d}}^{\mathrm{d}} \\
\left({ }^{\circ} \mathrm{C}\right)\end{array}$} \\
\hline & & & $\begin{array}{c}\text { In the } \\
\text { Feed }(\%)\end{array}$ & $\begin{array}{c}\text { In the } \\
\text { Copolymers }^{\mathrm{b}}(\%)\end{array}$ & & & \\
\hline P1 $\left(\mathrm{FO}_{3}\right.$-PT1 $)$ & 26,800 & 2.00 & 25 & 26.6 & 62 & 153 & 411 \\
\hline P2 $\left(\right.$ FO $_{1}$-PT1 $)$ & 14,600 & 1.55 & 50 & 48.6 & 88 & 175 & 401 \\
\hline P3 $\left(\mathrm{FO}_{3}\right.$-PT2 $)$ & 18,700 & 1.45 & 25 & 24.8 & 78 & 175 & 416 \\
\hline P4 (FO & 10,800 & 1.30 & 50 & 47.8 & 61 & 189 & 404 \\
\hline P5 $\left(\mathrm{FO}_{3}\right.$-PT3 $)$ & 49,600 & 2.40 & 25 & 26.1 & 82 & n.d. ${ }^{\mathrm{e}}$ & 410 \\
\hline P6 $\left(\right.$ FO $_{1}$-PT3 $)$ & 19,200 & 1.44 & 50 & 48.3 & 64 & n.d. ${ }^{\mathrm{e}}$ & 405 \\
\hline P7 $\left(\mathrm{FO}_{3}\right.$-PT4 $)$ & 59,200 & 2.65 & 25 & 25.9 & 86 & 130 & 415 \\
\hline P8 $\left(\right.$ FO $_{1}$-PT4 $)$ & 17,400 & 1.43 & 50 & 53.0 & 71 & 134 & 408 \\
\hline P9 $\left(\right.$ FO $_{3}$-PT5 $)$ & 102,900 & 3.68 & 25 & 27.0 & 87 & 132 & 419 \\
\hline 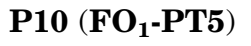 & 13,700 & 1.36 & 50 & 51.0 & 70 & 137 & 401 \\
\hline P11 $\left(\mathrm{FO}_{3}\right.$-PT6 $)$ & 30,200 & 1.63 & 25 & 25.5 & 89 & 158 & 409 \\
\hline P12 $\left(\right.$ FO $_{1}$-PT6 $)$ & 18,100 & 1.40 & 50 & 50.9 & 75 & n.d. ${ }^{\mathrm{e}}$ & 406 \\
\hline
\end{tabular}

a Molecular weights and polydispersity were measured by GPC, using THF as an eluent, polystyrene as a standard. $M_{\mathrm{n}}$ number-average molecular weight; $M_{\mathrm{w}}$, weight-average molecular weight.

${ }^{\mathrm{b}}$ Calculated from results of elemental analyses.

${ }^{\mathrm{c}}$ Glass transition temperature $\left({ }^{\circ} \mathrm{C}\right)$ was measured by DSC at a heating rate of $10{ }^{\circ} \mathrm{C} / \mathrm{min}$.

${ }^{\mathrm{d}}$ Temperature $\left({ }^{\circ} \mathrm{C}\right)$ at $5 \%$ weight loss measured by TGA at a heating rate of $20{ }^{\circ} \mathrm{C} / \mathrm{min}$ under nitrogen.

e No noticeable $T_{\mathrm{g}}$ was observed.

8400-27,900 and weight-average molecular weights $\left(M_{\mathrm{w}}\right)$ ranging $10,800-102900$, respectively, with polydispersity indices $\left(M_{\mathrm{w}} / M_{\mathrm{n}}\right)$ ranging 1.30-3.68. In contrast to $\mathbf{F O}_{\mathbf{3}}-\mathbf{P T}$ polymer derivatives (FO:PT $=3: 1$ ), the lower molecular weights of corresponding FO $_{\mathbf{1}}$-PT polymer analogues (FO:PT = 1:1) with higher PT contents were attributed to the lower solubilities of PT units (PT1-PT6) in their copolymerization processes. The PT contents of the resulting polymers can be estimated from elemental analysis (EA) results. It was found that $\mathrm{S}$ contents of the copolymers increased with the added PT contents during the copolymerization and were close to the monomer feed ratios of FO:PT (Table 1). ${ }^{19}$ For example, the actual molar ratios of PT/ FO were calculated from the $\mathrm{S}$ contents from the experimental EA divided by those from the calculated EA.

The thermal properties of the copolymers determined by thermogravimetric analysis (TGA) and DSC are shown in Table 1. The TGA thermograms of the D-A copolymers (P1-P12) revealed that $5 \%$ weight loss temperatures $\left(T_{\mathrm{d}} \mathrm{S}\right)$ were in the range of $401-416{ }^{\circ} \mathrm{C}$, indicative of excllent thermal stabilities. FO $_{\mathbf{1}}-\mathbf{P T}$ polymer derivatives (FO:PT $=1: 1$ ) showed lower $T_{\mathrm{d}}$ values than respective $\mathbf{F O}_{3}$-PT polymer analogues (FO:PT $=3: 1$, which may be probably due to the lower molecular weights of the former copolymers with lower FO contents. As for DSC properties, two thermally induced phase transitions, including a glass transition $\left(T_{\mathrm{g}}=158{ }^{\circ} \mathrm{C}\right)$ and a melting peak $\left(T_{\mathrm{m}}=180{ }^{\circ} \mathrm{C}\right)$, were merely observed in P11. However, P5, P6, and $\mathbf{P 1 2}$ exhibited no obvious phase transitions until $300{ }^{\circ} \mathrm{C}$ where slight decompositions began, and only one glass transition with $T_{\mathrm{g}}$ values between 130 and $189{ }^{\circ} \mathrm{C}$ were found in the other copolymers. Compared with $\mathbf{F O}_{\mathbf{3}}-\mathbf{P T}$ polymer derivatives (FO:PT = 3:1), FO $\mathbf{1}$-PT polymer analogues (FO:PT $=1: 1$ ) generally showed higher $T_{\mathrm{g}}$ values owing to the higher contents of rigid PT segments. These phenomena demonstrate that the physical properties of the copolymers, such as conformation, planarity, rigidity, and stacking, will be affected by the variation of PT structures and contents. Basically, the thermal stabilities of the copolymers are adequate for their applications in polymer solar cells and other optoelectronic devices.

The molecular structures of the copolymers were identified by FTIR and ${ }^{1} \mathrm{H}$ NMR. Representative FTIR spectra of copolymers P3, P4, P11, and $\mathbf{P 1 2}$ are shown in Figure 1, where the cyano functional groups appeared at 2270-2210 $\mathrm{cm}^{-1}$ in the triple bond region of FTIR spectra showed a sharp band with a medium intensity. As a result of the stretching modes of cyano groups in the copolymers, the absorption bands 


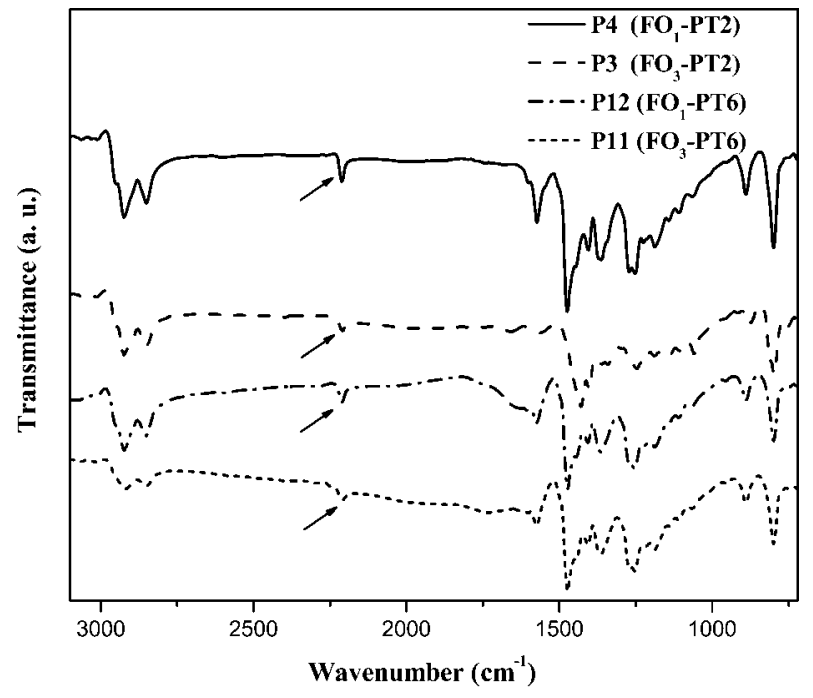

Figure 1. FTIR spectra of copolymers P3, P4, P11, and P12.

typically appeared at $2210 \mathrm{~cm}^{-1}$, and the intensity of this band increased with higher fractions of phenothiazine units in these polymers. Figure 2 shows the proton NMR spectra of monomer M4 and polymers P7-P8 in $\mathrm{CDCl}_{3}$. For example, the characteristic signal of the $\mathrm{CH}_{2}$ segments attached to the nitrogen atoms of phenothiazine units can be seen clearly ca. $\delta=3.8 \mathrm{ppm}$ for all monomers and copolymers. Compared with ${ }^{1} \mathrm{H}$ NMR spectra of polymers P7-P8 in Figure 2, it was generally found that sharper and more splitted signals of monomer M4 disappeared after polymerization. In addition, in contrast to polymer $\mathbf{P 7}$ (FO-PT4), a larger integrated signal (ca. $\delta=3.8 \mathrm{ppm}$ ) was observed in polymer P8 $\left(\right.$ FO $_{1}$-PT4) with a higher molar ratio of phenothiazine units (FO:PT4 = 1:1). Similar results were observed in the FTIR and ${ }^{1} \mathrm{H}$ NMR spectra for all copolymers.

\section{Optical Properties}

The photophysical characteristics of polymers P1-P12 were investigated by UV-vis absorption spectra in dilute THF solutions as well as in solid films, as revealed in Figures 3 and 4, respectively. The UV-vis absorption properties of the D-A copolymers based on 9,9-dihexylfluorene and phenothiazine units are presented in Table 2. As shown in Figure 3, most of the FOPT based copolymers (except P4 and P12 in THF solutions) exhibit two distinct peaks, and one of the peaks $\sim 375 \mathrm{~nm}$ is consistent with that reported for poly(9,9-dihexylfluorene) homo-

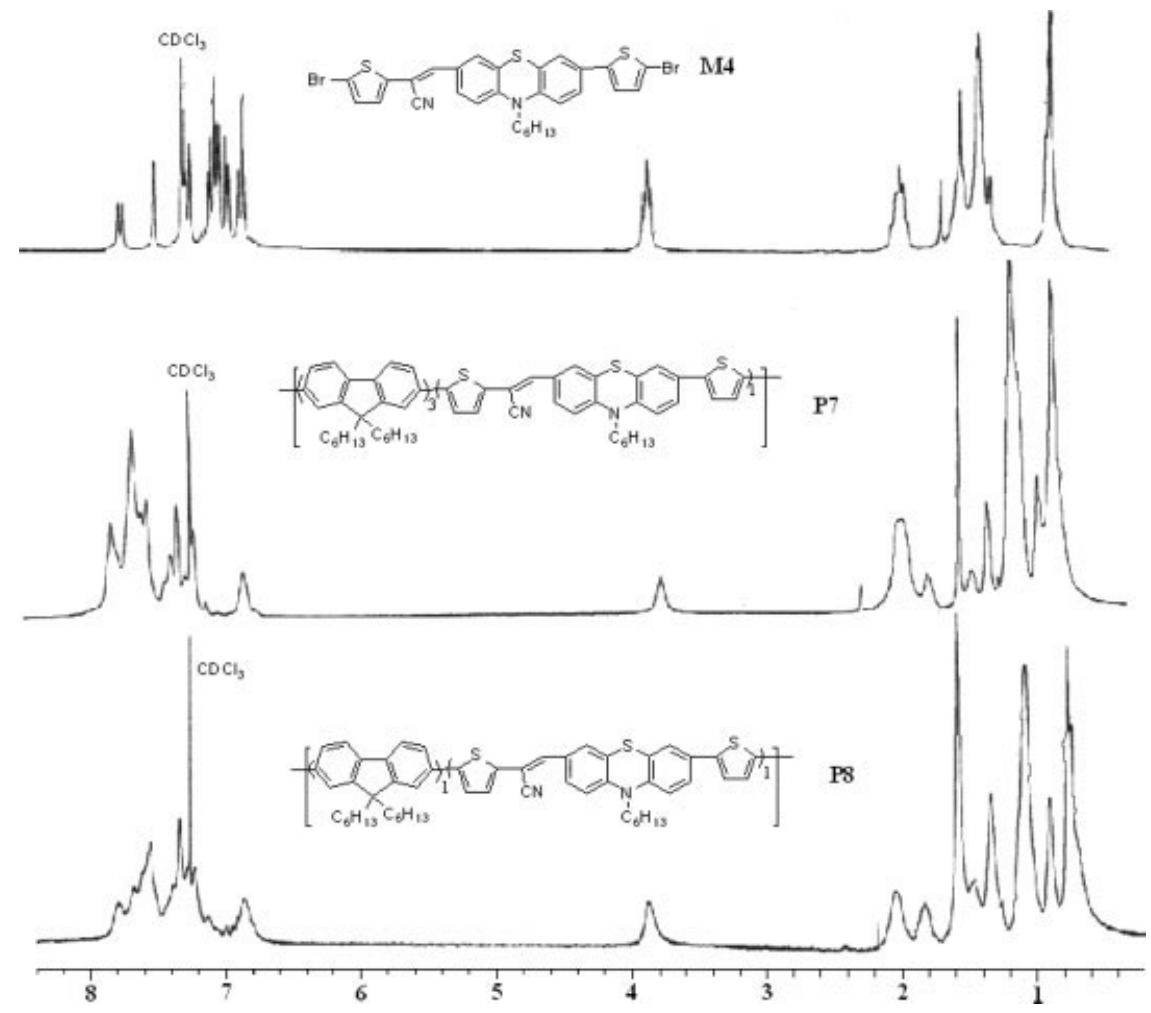

Figure 2. ${ }^{1} \mathrm{H}$ NMR spectra of monomer $\mathbf{M} 4$ and polymers P7-P8 in $\mathrm{CDCl}_{3}$. 

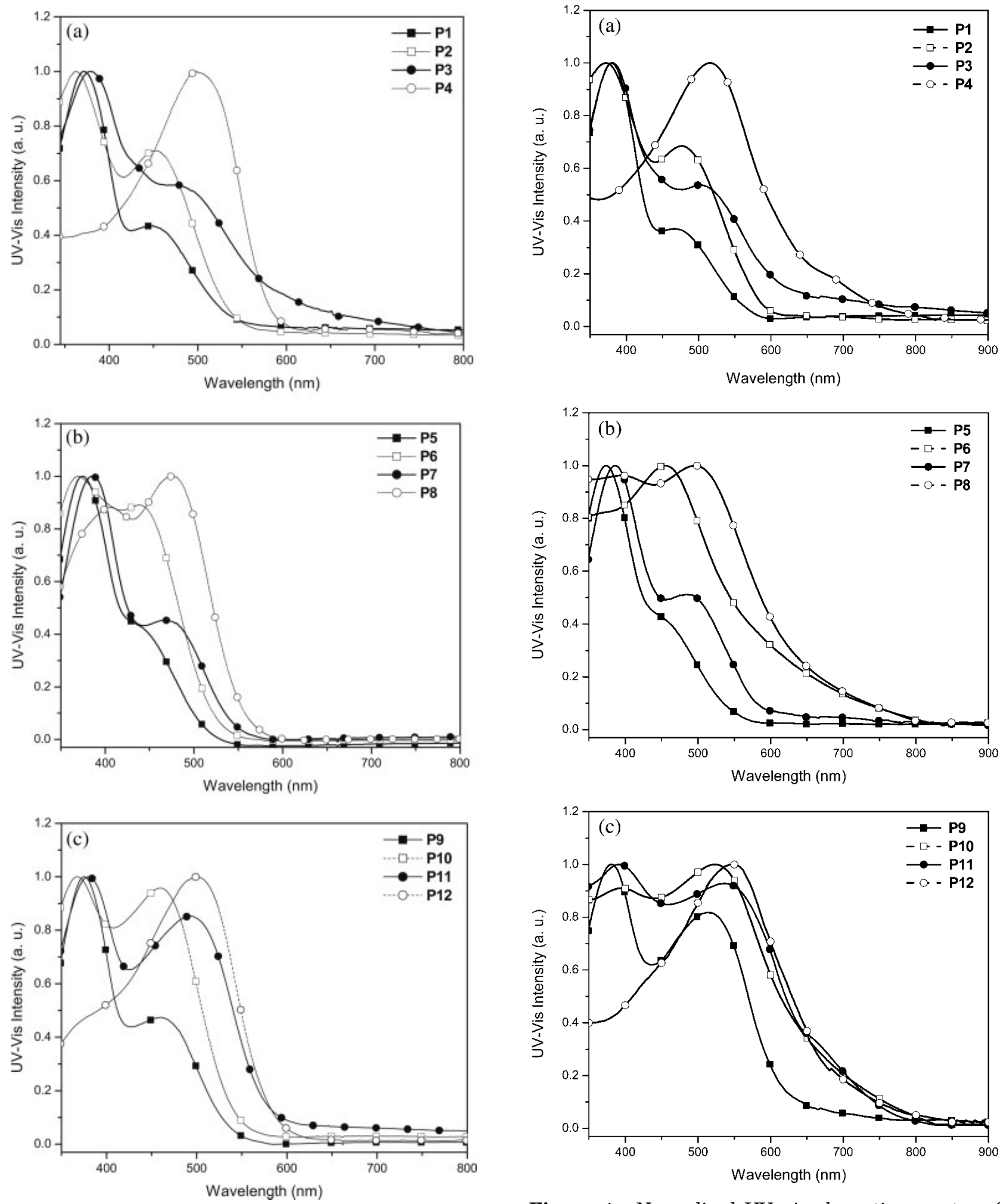

Figure 3. Normalized UV-vis absorption spectra of D-A copolymers in THF solutions $\left(\sim 10^{-6} \mathrm{M}\right)$ : (a) P1P4, (b) P5-P8, and (c) P9-P12.

Figure 4. Normalized UV-vis absorption spectra of D-A copolymers in solid films (spin-coating from chlorobenzene solutions): (a) P1-P4, (b) P5-P8, and (c) P9P12. 
Table 2. Optical Data of P1-P12 in THF Solutions $\left(\sim 10^{-6} \mathrm{M}\right)$ and Solid Films ${ }^{\mathrm{a}}$

\begin{tabular}{|c|c|c|c|c|c|c|c|}
\hline \multirow[b]{2}{*}{ Polymer } & \multicolumn{5}{|c|}{$\mathrm{UV}-\mathrm{vis}\left(\lambda_{\mathrm{a}}, \mathrm{nm}\right)$} & \multicolumn{2}{|c|}{$E_{\mathrm{g}}^{\mathrm{optb}}(\mathrm{eV})$} \\
\hline & Solution & $\lambda_{\text {edge }} \mathrm{c}$ & Film & $\lambda_{\text {edge }}{ }^{c}$ & $\Delta \lambda^{\mathrm{d}}$ & Solution & Film \\
\hline P1 $\left(\mathrm{FO}_{3}\right.$-PT1 $)$ & 373,450 & 560 & 375,472 & 600 & 22 & 2.21 & 2.06 \\
\hline P2 $\left(\right.$ FO $_{1}$-PT1 $)$ & 363,453 & 569 & 365,478 & 630 & 25 & 2.18 & 1.96 \\
\hline P3 $\left(\mathrm{FO}_{3}\right.$-PT2 $)$ & 378,480 & 592 & 381,510 & 700 & 30 & 2.09 & 1.77 \\
\hline P4 (FO & 491 & 600 & 516 & 830 & 25 & 2.07 & 1.49 \\
\hline P5 $\left(\mathrm{FO}_{3}\right.$-PT3 $)$ & 375,442 & 550 & 374,450 & 590 & 8 & 2.25 & 2.10 \\
\hline P6 $\left(\right.$ FO $_{1}-$ PT3 $)$ & 370,446 & 555 & 457 & 800 & 11 & 2.23 & 1.55 \\
\hline P7 $\left(\mathrm{FO}_{3}\right.$-PT4 $)$ & 380,475 & 570 & 388,490 & 690 & 15 & 2.18 & 1.79 \\
\hline P8 $\left(\right.$ FO $_{1}$-PT4 $)$ & 477 & 585 & 501 & 800 & 23 & 2.12 & 1.55 \\
\hline P9 $\left(\mathrm{FO}_{3}\right.$-PT5 $)$ & 376,460 & 560 & 380,518 & 715 & 58 & 2.21 & 1.73 \\
\hline 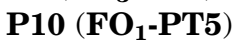 & 366,462 & 568 & 525 & 800 & 63 & 2.18 & 1.55 \\
\hline P11 $\left(\mathrm{FO}_{3}\right.$-PT6) & 380,493 & 608 & 390,540 & 800 & 47 & 2.04 & 1.55 \\
\hline P12 (FO & 500 & 613 & 548 & 800 & 48 & 2.02 & 1.55 \\
\hline
\end{tabular}

${ }^{\text {a }}$ Spin-coated from THF solution.

b The optical band gap was obtained from the equation $E_{\mathrm{g}}^{\mathrm{opt}}=1240 / \lambda_{\text {edge }}$

c The onset value of absorption spectrum in long wavelength direction.

${ }^{\mathrm{d}} \Delta \lambda=\lambda_{\text {max }}$ fiim $-\lambda_{\text {max } \text {,solution }}(\mathrm{nm})$.

polymer, ${ }^{43}$ which can be attributed to the fluorene segments in the copolymers. The other peak with an extra long wavelength absorption band appeared between 439 and $500 \mathrm{~nm}$, with tailing of the absorption to around $610 \mathrm{~nm}$ can be attributed to the PT units incorporated into the main-chains of the copolymers. For all copolymers, the higher PT contents in the random copolymers, the larger absorbance ratios of PT to fluorene units. However, a gradual blue shift of the short wavelength absorption accompanying with a small red shift of the long wavelength absorption were commonly observed in these polymers as the PT contents increased. This result can be explained by that as more PT segments were incorporated into the mainchains of the copolymers (with higher PT contents), more efficient conjugation or higher extents of aggregation occured between PT units but less sufficient conjugation or lower extents of aggregation arose between fluorene units. ${ }^{44-46}$ Two distinguished absorption features revealed that the electronic states of the two contents in random FO-PT copolymers were not well overlapped. Compared with the other random copolymers, P4 and P12 possessed completely different absorption spectra, where the absorption band $\sim 375 \mathrm{~nm}$ attributed to the fluorene segments disappeared and only a broad and strong absorption band showed $\sim 500 \mathrm{~nm}$. The single UV-vis absorption spectra of polymers $\mathbf{P 4}$ and $\mathbf{P 1 2}$ were quite different from the two sepa- rated absorptions of the other random copolymers derived from fluorene and low band-gap PT aromatic heterocyclics. ${ }^{47-49}$ This phenomenon suggested that the electronic configurations of both segments, i.e., fluorene and PT units, were somehow related to each other in the random copolymers P4 and P12.

The UV-vis absorption spectra of the copolymers in solid films are presented in Figure 4. The solid films showed similar absorption patterns as THF solutions (see Fig. 3). However, compared with solutions, the polymer films generally had $8-63 \mathrm{~nm}$ of red shifts due to the interchain association and aggregation in the solid state, and the spectral red-shifts were generally more significant when higher PT contents (FO-PT series in comparison with $\mathbf{F O}_{\mathbf{3}}$-PT series) and longer PT units (e.g., P9-P12 containing PT5 and PT6 units) were incorporated into the polymers (Table 2). According to UV absorptions of FO-PT copolymers in solid films, FO $_{1}$-PT polymer derivatives $(\mathrm{FO}: \mathrm{PT}=1: 1)$ possessed broader spectral absorption ranges (between 400 and $800 \mathrm{~nm}$ ) than their $\mathbf{F O}_{\mathbf{3}}$-PT polymer analogues (FO:PT $=3: 1$ ) to make them suitable candidates to harvest more photons.

As mentioned earlier in the reference, ${ }^{28}$ the UV-vis absorption result of $\mathbf{P 2}$ in solid films was similar to the reported data. Although some phenothiazine-based conjugated polymers have been developed, longer conjugation relationship of phenothiazine-based conjugated polymers 

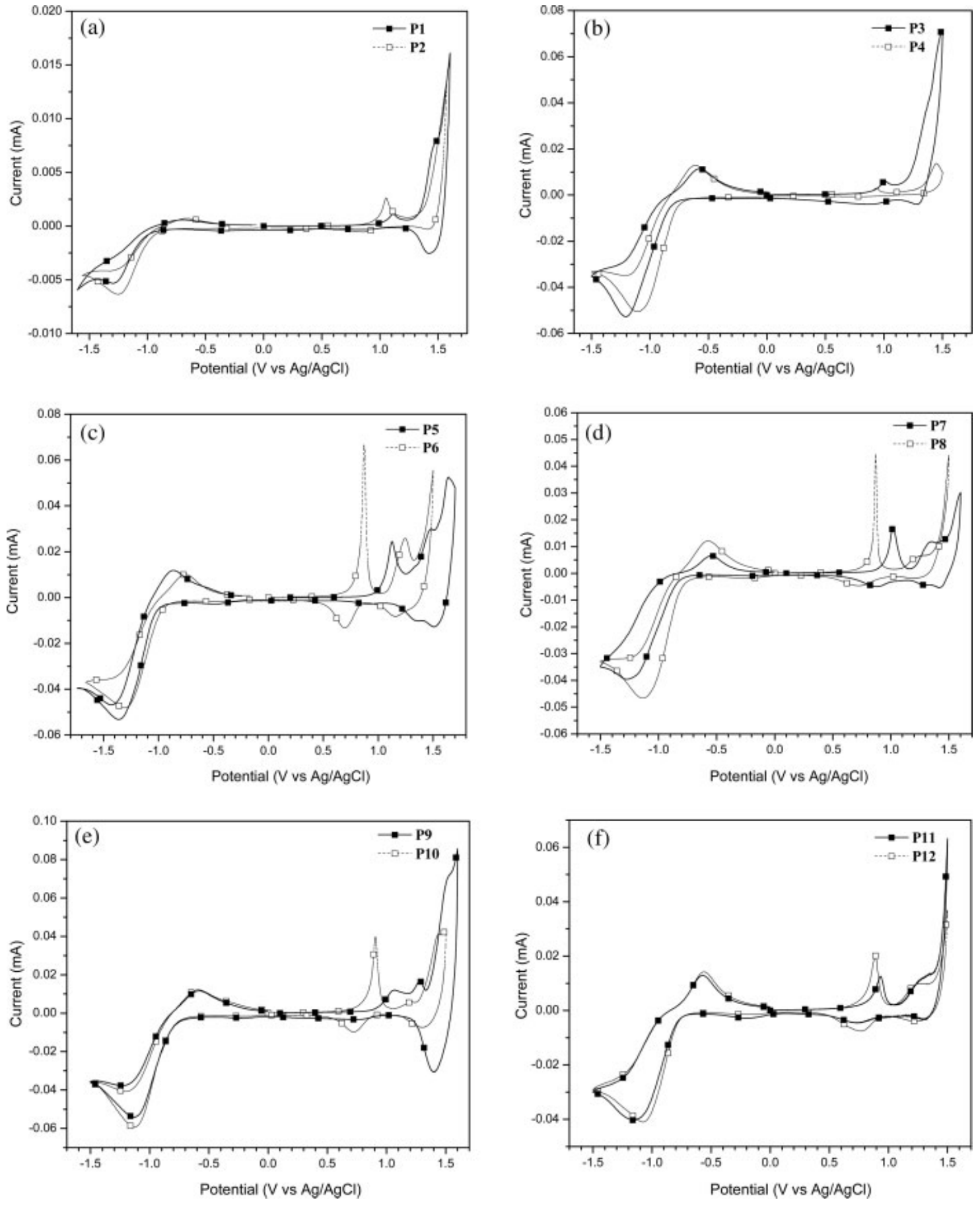

Figure 5. Cyclic voltammograms of fluorene-phenothiazine polymers P1-P12.

with aromatic (or heterocyclic) structures still need to be further explored. Therefore, our results can be compared with those reported ones and realize the effect of adding different numbers of aromatic rings (i.e., benzene and thiophene groups). ${ }^{28,40,50,51}$ Among these related literatures, Jenekhe and coworkers ${ }^{40}$ reported that the absorption maxima of the phenothiazine-fluorene alternating copolymer (PPTF) was located $\sim 384 \mathrm{~nm}$ in solid films. In our copolymers containing PT units, the donor (D) and acceptor (A) groups, such as thiophene and cyano groups, respectively, were incorporated into these polymers. Hence, the absorption max- 
Table 3. Electrochemical Potentials and Energy Levels of Copolymers P1-P12

\begin{tabular}{|c|c|c|c|c|c|c|c|c|}
\hline \multirow[b]{3}{*}{ Polymer } & \multirow{2}{*}{\multicolumn{2}{|c|}{$\frac{\text { Oxidation Potential }}{V \text { vs. } \mathrm{Ag} / \mathrm{Ag}^{+}}$}} & \multirow{2}{*}{\multicolumn{2}{|c|}{$\frac{\text { Reduction Potential }}{V \text { vs. } \mathrm{Ag} / \mathrm{Ag}^{+}}$}} & \multirow{2}{*}{\multicolumn{2}{|c|}{ Energy Level ${ }^{\mathrm{d}}(\mathrm{eV})$}} & \multirow{2}{*}{\multicolumn{2}{|c|}{ Bandgap (eV) }} \\
\hline & & & & & & & & \\
\hline & $E_{\text {ox/onset }}{ }^{\mathrm{b}}$ & $E_{\mathrm{ox} / \mathrm{o}}^{\mathrm{c}}$ & $E_{\text {red/onset }}{ }^{\mathrm{b}}$ & $E_{\mathrm{red} / \mathrm{o}}^{\mathrm{c}}$ & $E_{\text {номо }}$ & $E_{\text {LUMO }}$ & $E_{\mathrm{g}}^{\mathrm{ec}}$ & $E_{\mathrm{g}}^{\mathrm{opt}}$ \\
\hline P1 & 1.03 & 1.15 & -1.07 & -1.28 & -5.38 & -3.28 & 2.1 & 2.06 \\
\hline P2 & 1.00 & 1.06 & -0.99 & -1.19 & -5.35 & -3.36 & 1.99 & 1.96 \\
\hline P3 & 0.94 & 1.02 & -0.88 & -1.20 & -5.29 & -3.47 & 1.82 & 1.77 \\
\hline $\mathbf{P 4}$ & 0.83 & 0.94 & -0.86 & -1.10 & -5.18 & -3.49 & 1.69 & 1.49 \\
\hline P5 & 1.05 & 1.13 & -1.07 & -1.36 & -5.40 & -3.28 & 2.12 & 2.10 \\
\hline P6 & 0.80 & 0.87 & -1.04 & -1.31 & -5.15 & -3.31 & 1.84 & 1.55 \\
\hline P7 & 0.96 & 1.02 & -0.91 & -1.26 & -5.31 & -3.44 & 1.87 & 1.79 \\
\hline P8 & 0.75 & 0.87 & -0.89 & -1.13 & -5.10 & -3.46 & 1.64 & 1.55 \\
\hline P9 & 0.97 & 1.07 & -0.84 & -1.13 & -5.32 & -3.51 & 1.81 & 1.73 \\
\hline P10 & 0.74 & 0.91 & -0.83 & -1.13 & -5.09 & -3.52 & 1.57 & 1.55 \\
\hline P11 & 0.81 & 0.94 & -0.81 & -1.15 & -5.16 & -3.54 & 1.62 & 1.55 \\
\hline P12 & 0.78 & 0.89 & -0.81 & -1.08 & -5.13 & -3.54 & 1.59 & 1.55 \\
\hline
\end{tabular}

${ }^{\text {a }}$ Reduction and oxidation potentials measured by cyclic voltammetry in solid films.

b Onset oxidation and reduction potentials.

${ }^{\mathrm{c}}$ Formal oxidation and reduction potentials.

${ }^{\mathrm{d}} E_{\mathrm{HOMO}} / E_{\mathrm{LUMO}}=\left[-\left(E_{\text {onset }}-0.45\right)-4.8\right] \mathrm{eV}$ where $0.45 \mathrm{~V}$ is the value for ferrocene vs. $\mathrm{Ag} / \mathrm{Ag}^{+}$and $4.8 \mathrm{eV}$ is the energy level of ferrocene below the vacuum.

ima of FO-PT copolymers in solid films were around 450 to $548 \mathrm{~nm}$, which were further redshifted $\sim 66-164 \mathrm{~nm}$ compared with the corresponding spectrum of PPTF. The differences of the spectra between FO-PT and PPTF copolymer can be explained by the push-pull effect of $\mathrm{D}$ and $\mathrm{A}$ groups within the molecules and the increase of the effective conjugation lengths in the polymers. The optical band gaps of the copolymers in solid films, which were determined by the cutoff wavelengths of optical absorptions, are listed in Table 2. For all copolymers, the optical band gaps decreased with increasing PT contents and the push-pull (D-A) effect resulted in narrower optical band gaps of copolymers by our approach, which induced broader visible absorption ranges (between 400 and $800 \mathrm{~nm}$ ) than PPTF.

\section{Electrochemical Characterization}

The electronic states, i.e., highest occupied molecular orbital (HOMO) and lowest unoccupied molecular orbital (LUMO) levels, of the FO-PT copolymers were investigated by cyclic voltammetry (CV) to understand the charge injection processes in these new NBG polymers and their PVC devices. The oxidation and reduction $\mathrm{CVs}$ of polymers P1-P12 in solid films are displayed in Figure 5. The electrochemical measurements of the formal potentials, onset potentials, band gaps, and the estimated positions of the upper edges of the valence band (HOMO) and the lower edges of the conduction band (LUMO) are summarized in Table $3 . \mathrm{Ag} / \mathrm{AgCl}$ was served as a reference electrode, and it was calibrated by ferrocene $\left(E_{\text {ferrocene }}^{1 / 2}=0.45 \mathrm{mV}\right.$ versus $\left.\mathrm{Ag} / \mathrm{AgCl}\right)$. The HOMO and LUMO energy levels were estimated by the oxidation and reduction potentials from the reference energy level of ferrocene (4.8 $\mathrm{eV}$ below the vacuum level) according to the following equation ${ }^{52,53}: E_{\mathrm{HOMO} / \mathrm{LUMO}}=\left[-\left(E_{\text {onset }}-\right.\right.$ $0.45)-4.8] \mathrm{eV}$. For all copolymers, two $p$-doping processes and one $n$-doping process could be recorded, and partial reversibilities in both $p$ doping and $n$-doping processes were evidenced from the areas and close proximity of the anodic and cathodic scans. The formal oxidation and reduction potentials of P1-P12 were in the range of (0.87-1.15) $\mathrm{eV}$ and -(1.08-1.36) $\mathrm{eV}$, respectively. The band-gap values directly measured by $\mathrm{CV}$ ( $E_{\mathrm{g}}^{\mathrm{ec}}$ between 1.57 and $2.12 \mathrm{eV}$ ) and the optical band-gap values observed from UVvis spectra $\left(E_{\mathrm{g}}^{\mathrm{opt}}\right.$ between 1.55 and $\left.2.10 \mathrm{eV}\right)$ were close to each other.

Two different onsets of oxidation processes occurred between $\sim 0.74-1.05$ and $1.28-1.35 \mathrm{eV}$, which were originated from the $\mathrm{PT}$ and $\mathrm{FO}^{53}$ segments, respectively, in FO-PT copolymers. The onsets of the reduction processes of all 
copolymers were observed at $-(0.81-1.07) \mathrm{eV}$, except the data of copolymer P2, which is different from the earlier reported result. ${ }^{28}$ Because the reduction potential of polyfluorene homopolymer was observed typically at $-2.28 \mathrm{eV}^{53}$ the moderate reduction wave at $-(0.81-1.07) \mathrm{eV}$ should be attributed to the electron withdrawing effect of the PT moiety in the reduction process. The second reduction wave corresponding to the fluorene segment was unable to record. It could be probably because the reduced states of the copolymers at higher negative voltages were unstable. According to the previous equation, the copolymers were relatively stable up to oxidation with low HOMO levels varying 5.09-5.40 $\mathrm{eV}$. Owing to the results of low HOMO levels, the copolymers were easily handled in air without encountering undesired oxidation. The copolymers possessing LUMO levels $\sim 3.28-3.54$ $\mathrm{eV}$ were also good donors for charge transfer to PCBM acceptors (with 0.66-0.92 eV LUMO offsets regarding LUMO level of PCBM being at $4.2 \mathrm{eV}$ ). These characters are valuable properties to make use of these materials into optoelectronics.

Among the reports of phenothiazine homopolymer (PHPT) and PPTF copolymer, it is clear that HOMO levels of the polymers were dominated by the contribution from the phenothiazine moiety, but no reduction wave of either polymer was observed for LUMO levels. ${ }^{40}$ Interestingly, there are clear differences of $n$-doping processes between the previous phenothiazine copolymers without any functional groups and our PT copolymers (with detectable reduction waves). Furthermore, the presence of different numbers of electron-donating thiophenes and electron-withdrawing cyano groups in both sides of our phenothiazine moieties changed the electrical properties of PT copolymers so that they possessed both $p$-type and $n$-type properties. Therefore, it is clear that the HOMO/LUMO levels of FO-PT copolymers have been significantly varied relative to those of PFO and PHPT due to the modulated push-pull strengths of thiophene and cyano groups. Another important feature was also observed that as the PT contents of the copolymers were equivalent to 25 and $50 \%$, the first onset potentials of $p$-doping and $n$-doping waves decreased with increasing PT contents. This result eventually induced narrower band-gaps of FO-PT copolymers owing to the increase of their HOMO levels and the reduction of their LUMO levels. The characters of reversible electrochemical oxidation and reduction processes in the copolymers suggested promising prospects for superior electrochemical stabilities in the applications of organic electronic devices.

\section{Polymeric Photovoltaic Cell (PVC) Properties}

In the fabrication of bulk-heterojunction photovoltaic cell (PVC) devices, copolymers P2, P6, P8, P10, and P12 were used as the donor phase to blend with different ratios of methanofullerene6,6-PCBM as the typical acceptor phase. As described by UV absorptions of FO-PT copolymers in solid films, FO $\mathbf{F}-\mathbf{P T}$ polymer derivatives $(\mathrm{FO}: \mathrm{PT}=1: 1)$ possessed broader spectral absorption coverages than their $\mathbf{F O}_{\mathbf{3}}$-PT polymer analogues (FO:PT $=3: 1$ ) in the visible ranges between 400 and $800 \mathrm{~nm}$. Because of the benefits of narrower band-gaps and broader visible absorption ranges in $\mathbf{F O} \mathbf{O}_{\mathbf{1}}$-PT polymers with higher PT contents, $\mathbf{F O}_{\mathbf{1}}$-PT polymer derivatives were chosen to survey their potentials for PVC applications. In Figure 6(a), the HOMO and LUMO levels of $\mathbf{F O}_{\mathbf{1}}-\mathbf{P T}$ polymer derivatives also match those of good hole-transporting materials for PVC devices with an electron-transporting material PCBM. Thus, FO $\mathbf{F}-\mathbf{P T}$ polymer derivatives were appropriate for the fabrication of PVC devices with a configuration of ITO/ PEDOT:PSS/FO $\mathbf{1}$-PT:PCBM/LiF/Al as shown in

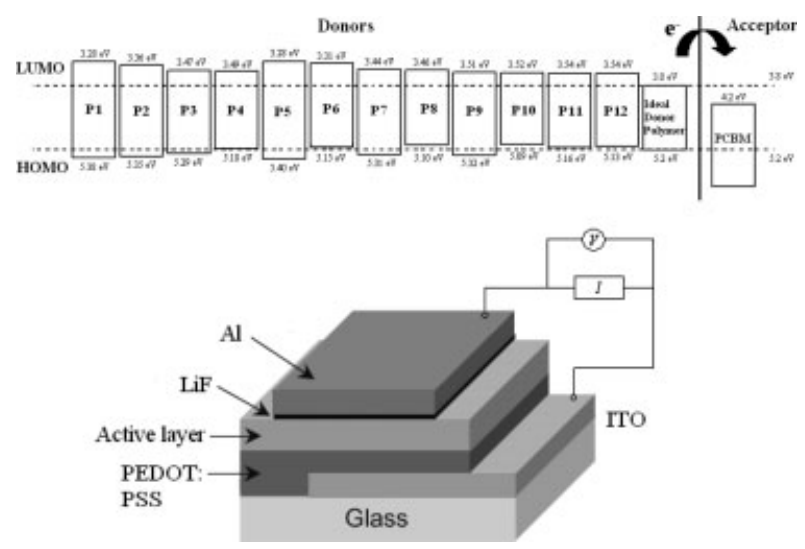

Figure 6. (a) Energy levels for an ideal donor polymer for PCBM along with donors P1-P12. Dashed lines display the HOMO and LUMO thresholds of an ideal donor polymer between $5.2-3.8 \mathrm{eV}$ for air stability $(5.2 \mathrm{eV})$ and effective charge transfer to PCBM $(3.8 \mathrm{eV}) .^{54}$ (b) Device structure consisting of an 100 $\mathrm{nm}$ thick blending active layer (copolymers:PCBM), which was sandwiched between PEDOT:PSS and an aluminum top electrode. 


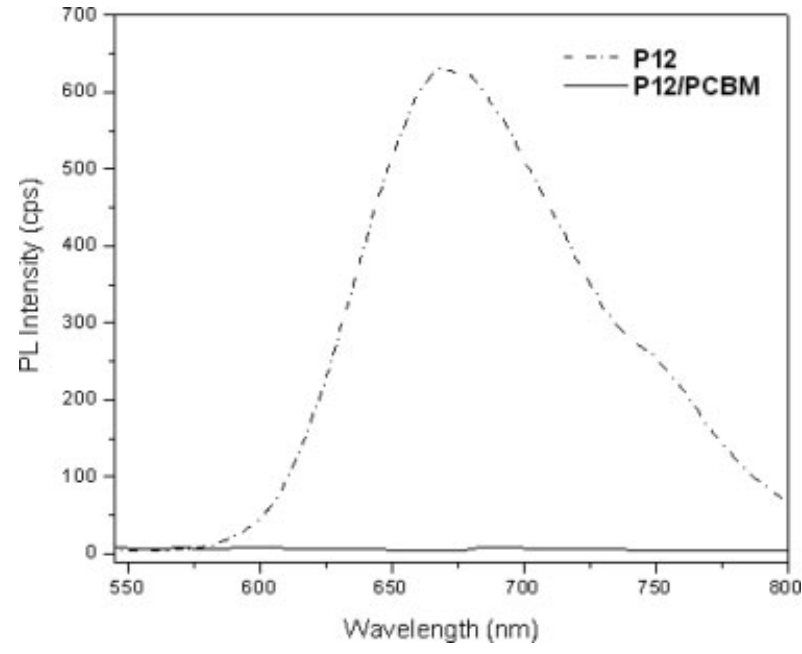

Figure 7. PL spectra of $\mathbf{P 1 2}$ film and a blending film of P12/PCBM (1:4 w/w).

Figure 6(b). To evaluate the PVC properties of FO-PT copolymers, a composite thin film of FO - -PT:PCBM was prepared by spin-coating a solution of P12 and PCBM (1:4 w/w) in the mixture solution of chlorobenzene and chloroform (1:1 vol) onto a quartz plate, and its PL spectrum was recorded, as shown in Figure 7. Compared with the PL spectrum of pure P12, complete PL quenching was observed as a result of blending P12 with PCBM, which could be attributed to the different kinetics of charge transfer $\left(\sim 10^{-14} \mathrm{~s}\right)$ and recombination $\left(\sim 10^{-3}\right.$ s). ${ }^{31}$ The PL quenching property indicates that

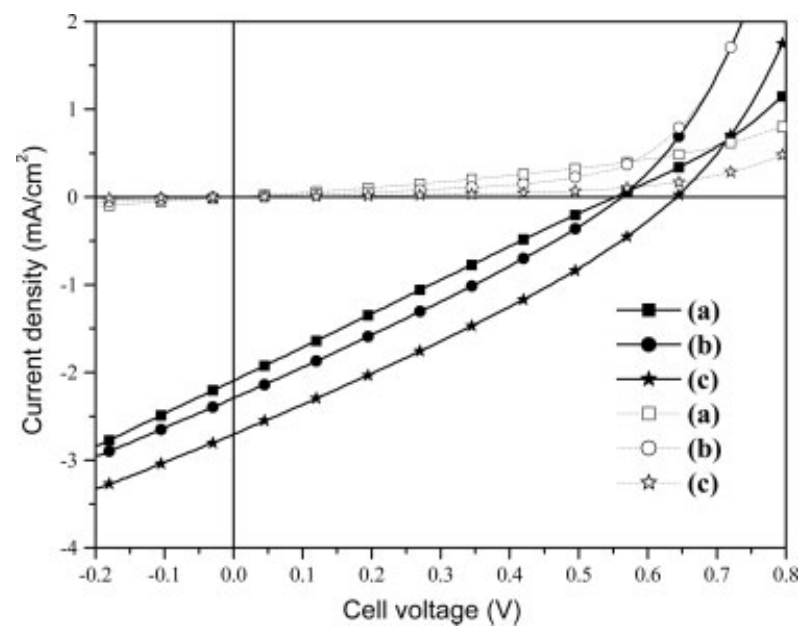

Figure 8. $I-V$ curves of the polymer solar cells with different compositions of P12/PCBM (a) 1:1 w/w (square symbols), (b) 1:2 w/w (circle symbols), and (c) $1: 4 \mathrm{w} / \mathrm{w}$ (star symbols) measured in the dark (dash lines) and under the illumination of AM 1.5, $100 \mathrm{~mW} /$ $\mathrm{cm}^{2}$ (solid lines).
FO-PT copolymers can be used as proper electron donors in PVC devices.

The $I-V$ characteristics of photovoltaic cell devices with different weight ratios of P12:PCBM $=1: 1,1: 2$, and 1:4 as an active layer are presented in Figure 8, which were measured under AM 1.5 illumination for a calibrated solar simulator with an intensity of $100 \mathrm{~mW} / \mathrm{cm}^{2}$. The photovoltaic properties obtained from the $I-V$ curves are listed in Table 4. The open circuit voltage $\left(V_{\mathrm{oc}}\right)$, short circuit current $\left(I_{\mathrm{sc}}\right)$, and fill factor (FF) of the PVC device based on the ratio of P12:PCBM = 1:4 (w/w) were $0.64 \mathrm{~V}, 2.7 \mathrm{~mA} /$ $\mathrm{cm}^{2}$, and $29 \%$, respectively, which were all higher than those of PVC devices based on the ratios of P12:PCBM = 1:1 and 1:2 (w/w). Generally, the values of ECE in PVC devices are sensitive to the weight ratios of acceptors to donors. In the case of P12/PCBM blends, the best efficiency observed was P12:PCBM $=1: 4(\mathrm{w} / \mathrm{w})$, which was a similar dependence on donor/ acceptor weight ratio in some earlier publications. ${ }^{16,47,55}$ In addition, according to the photovoltaic results of these copolymers in Table 4, the highest ECE value of $0.51 \%$ was obtained from a solar cell device with P12 as an electron donor. Apparently, comparing the molecular structures of copolymers P2, P6, P8, P10, and $\mathbf{P 1 2}$, the longest conjugation length and the heterocyclic structures (thiophene units) of $\mathbf{P 1 2}$ could lead to the highest photovoltaic efficiency among these synthesized copolymers. This result indicates that the incorporation of longer conjugation lengths and heterocyclic moieties into conjugated copolymers could make favorable contribution to photovoltaic properties.

Several parameters are suspected to responsible for the low efficiencies in the PVCs, such as

Table 4. Photovoltaic Properties of Copolymers with a Solar Cell Device Configuration of ITO/ PEDOT:PSS/Copolymer: PCBM/LiF/Al ${ }^{\mathrm{a}}$

\begin{tabular}{lcccc}
\hline Active Layer & $\begin{array}{c}V_{\text {oc }} \\
(\mathrm{V})\end{array}$ & $\begin{array}{c}I_{\text {sc }} \\
\left(\mathrm{mA} / \mathrm{cm}^{2}\right)\end{array}$ & $\begin{array}{c}\mathrm{FF} \\
(\%)\end{array}$ & $\begin{array}{c}\mathrm{ECE} \\
(\%)\end{array}$ \\
\hline P2/PCBM(1:4) & 0.43 & 1.86 & 27 & 0.22 \\
P6/PCBM(1:4) & 0.52 & 1.46 & 22 & 0.17 \\
P8/PCBM(1:4) & 0.27 & 2.21 & 27 & 0.16 \\
P10/PCBM(1:4) & 0.53 & 1.30 & 26 & 0.18 \\
P12/PCBM(1:1) & 0.55 & 2.10 & 25 & 0.29 \\
P12/PCBM(1:2) & 0.56 & 2.30 & 28 & 0.36 \\
P12/PCBM(1:4) & 0.64 & 2.70 & 29 & 0.51 \\
\hline \multicolumn{5}{c}{ a Measured under AM 1.5 irradiation, $100 \mathrm{~mW} / \mathrm{cm}}$.
\end{tabular}


the thickness of the film, the disorder of the film morphology, and the large difference of chargecarrier mobility, etc. The lower molecular weights of $\mathbf{F O}_{\mathbf{1}}-\mathbf{P T}$ polymer derivatives resulted in the solid films with thinner thicknesses and the fewer harvested photons from the solar energy. Therefore, the $I_{\mathrm{sc}}$ values of the copolymers (listed in Table 4) were only $1.30-2.70 \mathrm{~mA} /$ $\mathrm{cm}^{2}$. For example, the $V_{\mathrm{oc}}, I_{\mathrm{sc}}, \mathrm{FF}$, and ECE values of P2 were $0.43 \mathrm{~V}, 1.86 \mathrm{~mA} / \mathrm{cm}^{2}, 27 \%$, and $0.22 \%$, respectively, which were not as high as the same polymer published earlier by Cho and coworkers, ${ }^{47-49}$ which might be due to the lower molecular weight or the lower FF of P2. In comparison with the photovoltaic results of P2, P6, P8, and P10, the PVC based on P12 showed a much higher $V_{\text {oc }}$ value of $0.64 \mathrm{~V}$ in Table 4, except $\mathbf{P 4}$ because of its poor solubility. Generally, the $V_{\text {oc }}$ value is related to the difference between the oxidation potential of the donor and the reduction potential of the acceptor (PCBM). ${ }^{56}$ However, compared with the other polymers with lower HOMO levels, the PVC based on $\mathbf{P 1 2}$ possessing the highest $V_{\text {oc }}$ value did not follow the previous general regulation in this work. In addition to the above-mentioned influences on PVCs, the deficits of the ECE values in our polymers were mainly caused by low FFs which indicate lacks of ordered continuity in the polymer/PCBM blends. ${ }^{57,58}$ The disorder of the film morphology also severely affects the charge carrier mobility, which is believed to be the bottleneck for the $I_{\mathrm{sc}}$ values. ${ }^{58}$

Although the photovoltaic properties of the copolymers in this work were not the best results compared with the other low bandgap polymers, the preliminary results of PVC devices made of the newly synthesized polymers were still not optimized. Further improvements are underway to optimize the PVC devices by the modification of the film morphology, layer thicknesses, postproduction treatment conditions, and the other electron acceptors.

\section{CONCLUSIONS}

Souble conjugated donor-acceptor low-band-gap copolymers derived from 9,9-dihexylfluorene (FO) and phenothiazine-arylcyanovinyl units were synthesized by palladium (0)-catalyzed Suzuki coupling reactions and characterized by NMR, FTIR, and elemental analyses. The more heterocyclic units and cyano-groups incorpo- rated into phenothiazine derivatives, the stronger strength of intramolecular charge-transfer interaction. Thus, the optical and electrochemical properties of the copolymers were induced to visible and even further to near infrared absorption with narrow band gaps, which the lowest result were $1.55 \mathrm{eV}$. Photoluminescence quenching measurements indicated that FO-PT was functioning as a photo-excited donor in case of blending with PCBM. Bulk heterojunction PVC devices fabricated from a thin film composed of a blend of FO $_{1}$-PT polymer derivatives and PCBM, with the configuration of ITO/PEDOT: $\mathrm{PSS} /\left(\mathbf{F O}_{1}\right.$-PT:PCBM $\left.=1: 4\right) / \mathrm{LiF} / \mathrm{Al}$, showed the preliminary results of the PVC devices, and their optoelectronic performance can also be much improved in the future.

The authors thank the financial support from the National Science Council of Taiwan (ROC) through NSC 94-2113M-009-011 and Chung-Shan Institute of Science and Technology (in Taiwan).

\section{REFERENCES AND NOTES}

1. Winder, N. S.; Saridifti, N. S. J Mater Chem 2004, 14, 1077-1086.

2. Günes, S.; Neugebauer, H.; Sariciftci, N. S. Chem Rev 2007, 107, 1324-1338.

3. Yang, X.; Loos, J. Macromolecules 2007, 40, 1353-1362.

4. Koppe, M.; Scharber, M.; Brabec, C.; Duffy, W.; Heeney, M.; McCulloch, I. Adv Funct Mater 2007, 17, 1371-1376.

5. Hou, J.; Tan, Z.; Yan, Y.; He, Y.; Yang, C.; Li, Y. J Am Chem Soc 2006, 128, 4911-4916.

6. Peet, J.; Kim, J. Y.; Coates, N. E.; Ma, W. L.; Moses, D.; Heeger, A. J.; Bazan, G. C. Nat Mater 2007, 6, 497-500.

7. Mülhlbacher, D.; Scharber, M.; Morana, M.; Zhu, Z.; Waller, D.; Gaudiana, R.; Brabec, C. Adv Mater 2006, 18, 2884-2889.

8. Zhu, Z.; Waller, D.; Gaudiana, R.; Morana, M.; Mülhlbacher, D.; Scharber, M.; Brabec, C. Macromolecules 2007, 40, 1981-1986.

9. Huo, L.; He, C.; Han, M.; Zhou, E.; Li, Y. F. J Polym Sci Part A: Polym Chem 2007, 45, 3861-3871.

10. Colladet, K.; Fourier, S.; Cleij, T. J.; Lutsen, L.; Gelan, J.; Vanderzande, D. Macromolecules 2007, 40, 65-72.

11. Shahid, M.; Ashraf, R. S.; Klemm, E.; Sensfuss, S. Macromolecules 2006, 39, 7844-7853.

12. Zhan, X.; Tan, Z.; Domercq, B.; An, Z.; Zhang, X.; Barlow, S.; Li, Y.; Zhu, D.; Kippelen, B.; Marder, S. R. J Am Chem Soc 2007, 129, 7246-7247. 
13. Zhu, Y.; Champion, R. D.; Jenekhe, S. A. Macromolecules 2006, 39, 8712-8719.

14. Champion, R. D.; Cheng, K. F.; Pai, C. L.; Chen, W. C.; Jenekhe, S. A. Macromol Rapid Commun 2005, 26, 1835-1840.

15. Shi, C.; Yao, Y.; Yang, Y.; Pei, Q. J Am Chem Soc 2006, 128, 8980-8986.

16. Hou, Q.; Zhou, Q.; Zhang, Y.; Yang, W.; Yang, R.; Cao, Y. Macromolecules 2004, 37, 6299-6305.

17. Shin, W. S.; Kim, S. C.; Lee, S. J.; Jeon, H. S.; Kim, M. K.; Daidu, B. V. K.; Jin, S. H.; Lee, J. K.; Lee, J. W.; Gal, Y. S. J Polym Sci Part A: Polym Chem 2007, 45, 1394-1402.

18. Ashraf, R. S.; Hoppe, H.; Shahid, M.; Gobsch, G.; Sensfuss, S.; Klemm, E. J Polym Sci Part A: Polym Chem 2006, 44, 6952-6961.

19. Xia, Y.; Luo, J.; Deng, X.; Li, X.; Li, D.; Zhu, X.; Yang, W.; Cao, Y. Macromol Chem Phys 2006, 207, 511-520.

20. Turkarslan, O.; Ak, M.; Tanyeli, C.; Akhmedov, I. M.; Toppare, L. J Polym Sci Part A: Polym Chem 2007, 45, 4496-4503.

21. Thompson, B. C.; Kim, Y. G.; McCarley, T. D.; Reynolds, J. R. J Am Chem Soc 2006, 128, 12714-12725.

22. Zhang, F.; Mammo, W.; Andersson, L. M.; Admassie, S.; Andersson, M. R.; Inganäs, O. Adv Mater 2006, 18, 2169-2173.

23. van Mullekom, H. A. M.; Vekemans, J. A. J. M.; Havinga, E. E.; Meijer, E. W. Mater Sci Eng 2001, $32,1-40$.

24. Shen, Z.; Strauss, J.; Daub, J.; Chem Commun 2002, 460-461.

25. Lai, R. Y.; Kong, X.; Jenekhe, S. A.; Bard, A. J. J Am Chem Soc 2003, 125, 12631-12639.

26. Liu, Y.; Cao, H.; Li, J.; Chen, Z.; Cao, S.; Xiao, L.; $\mathrm{Xu}$, S.; Gong, Q. J Polym Sci Part A: Polym Chem 2007, 45, 4867-4878.

27. Yang, L. Y.; Wang, C.; Li, L. Q.; Janietz, S.; Wedel, A.; Hua, Y. L.; Yin, S. G. J Polym Sci Part A: Polym Chem 2007, 45, 4291-4299.

28. Cho, N. S.; Park, J. H.; Lee, S. K.; Lee, J.; Shim, H. K. Macromolecules 2006, 39, 177-183.

29. Tang, W.; Kietzke, T.; Vemulamada, P.; Chen, Z. K. J Polym Sci Part A: Polym Chem 2007, 45, 5266-5276.

30. Zou, Y.; Wu, W.; Sang, G.; Yang, Y.; Liu, Y.; Li, Y. Macromolecules 2007, 40, 7231-7237.

31. Roncali, J. Chem Rev 1997, 97, 173-206.

32. Tu, G.; Li, H.; Forster, M.; Heiderhoff, R.; Balk, L. J.; Scherf, U. Macromolecules 2006, 39, 4327-4331.

33. Lee, J.; Cho, N. S.; Lee, J.; Lee, S. K.; Shim, H. K. Synth Met 2005, 155, 73-79.

34. Ranger, M.; Rondeau, D.; Leclerc, M. Macromolecules 1997, 30, 7686-7691.

35. Liu, B.; Yu, W. L.; Lai, Y. H.; Huang, W. Chem Mater 2001, 13, 1984-1991.
36. Jin, S. H.; Kim, M. Y.; Koo, D. S.; Kim, Y. I. Chem Mater 2004, 16, 3299-3307.

37. Lim, E.; Jung, B. J.; Shim, H. K. Macromolecules 2003, 36, 4288-4293.

38. Cho, N. S.; Hwang, D. H.; Jung, B. J.; Lim, E.; Lee, J.; Shim, H. K. Macromolecules 2004, 37, 52655273.

39. Yeh, H. C.; Chan, L. H.; Wu, W. C.; Chen, C. T. J.; Mater Chem 2004, 14, 1293-1298.

40. Kong, X.; Kulkarni, A. P.; Jenekhe, S. A. Macromolecules 2003, 36, 8992-8999.

41. Liu, Y.; Li, J.; Cao, H.; Qu, B.; Chen, Z.; Gong, Q.; Xu, S.; Cao, S. Polym Adv Technol 2006, 17, 468-473.

42. Tolman, C. A.; Seidel, W. C.; Gerlach, D. H. J Am Chem Soc 1972, 94, 2669-2676.

43. Kulkarni, A. P.; Jenekhe, S. A. Macromolecules 2003, 36, 5285-5296.

44. Cao, D.; Liu, Q.; Zeng, W.; Han, S.; Peng, J.; Liu, S. Macromolecules 2006, 39, 8347-8355.

45. Huang, F.; Hou, L.; Wu, H.; Wang, X.; Shen, H.; Cao, W.; Yang, W.; Cao, Y. J Am Chem Soc 2004, 126, 9845-9853.

46. Cho, N. S.; Hwang, D. H.; Lee, J. I.; Jung, B. J.; Shim, H. K. Macromolecules 2002, 35, 1224-1228.

47. Wang, F.; Luo, J.; Yang, K.; Chen, J.; Huang, F.; Cao, Y. Macromolecules 2005, 38, 2253-2260.

48. Yang, J.; Jiang, C.; Zhang, Y.; Yang, R.; Yang, W.; Hou, Q.; Cao, Y. Macromolecules 2004, 37, 12111218.

49. Svensson, M.; Zhang, F.; Veenstra, S. C.; Verhees, W. J. H.; Hummelen, J. C.; Kroon, J. M.; Inganas, O.; Andersson, M. R. Adv Mater 2003, 15, 988991.

50. Park, J. H.; Cho, N. S.; Jung, Y. K.; Cho, H. J.; Shim, H. K.; Kim, H.; Lee, Y. S. Org Electron 2007, 8, 272-285.

51. Lee, J.; Lee, J. I.; Park, M. J.; Jung, Y. K.; Cho, N. S.; Cho, H. J.; Hwang, D. H.; Lee, S. K.; Park, J. H.; Chu, H. Y.; Shim, H. K. J Polym Sci Part A: Polym Chem 2007, 45, 1236-1246.

52. Chen, Y. Y.; Tao, Y. T.; Lin, H. C. Macromolecules 2006, 39, 8559-8566.

53. Janietz, S.; Bradley, D. D.C.; Grelly, M.; Giebeler, C.; Inbasekaran, M.; Woo, E. P. Appl Phys Lett 1998, 73, 2453-2455.

54. Saricitfci, N. S.; Smilowitz, L.; Hegger, A. J.; Wudl, F. Science 1992, 258, 1474-1476.

55. Pommerehne, J.; Vestweber, H.; Guss, W.; Mahrt, R. F.; Bassler, H.; Porsch, M.; Daub, J. Adv Mater 1995, 7, 551-554.

56. Yang, R.; Tian, R.; Yan, J.; Zhang, Y.; Yang, J.; Hou, Q.; Yang, W.; Zhang, C.; Cao, Y. Macromolecules 2005, 38, 244-253.

57. Koster, L. J. A.; Mihailetchi, V. D.; Blom, P. W. M. Appl Phys Lett 2006, 88, 093511.

58. Coffey, D. C.; Reid, O. G.; Rodovsky, D. B.; Bartholomew, G. P.; Ginger, D. S. Nano Lett 2007, 7, 738-744. 